\title{
Upregulation of the actin cytoskeleton via myocardin leads to increased expression of type 1 collagen
}

\author{
Zengdun Shi and Don C Rockey
}

Liver fibrosis, a model wound healing system, is characterized by excessive deposition of extracellular matrix (ECM) in the liver. Although many fibrogenic cell types may express ECM, the hepatic stellate cell (HSC) is currently considered to be the major effector. HSCs transform into myofibroblast-like cells, also known as hepatic myofibroblasts in a process known as activation; this process is characterized in particular by de novo expression of smooth muscle alpha actin (SM a-actin) and type 1 collagen. The family of actins, which form the cell's cytoskeleton, are essential in many cellular processes. $\beta$-actin and cytoplasmic $\gamma$-actin ( $\gamma$-actin) are ubiquitously expressed, whereas SM a-actin defines smooth muscle cell and myofibroblast phenotypes. Thus, SM a-actin is tightly associated with multiple functional properties. However, the regulatory mechanisms by which actin isoforms might regulate type 1 collagen remain unclear. In primary HSCs from normal and fibrotic rat liver, we demonstrate that myocardin, a canonical SRF cofactor, is upregulated in hepatic myofibroblasts and differentially regulates SM a-actin, $\gamma$-actin, and $\beta$-actins through activation of an ATTA box in the SM $\alpha$-actin and a CCAAT box in $\gamma$-actin and $\beta$-actin promoters, respectively; moreover, myocardin differentially activated serum response factor (SRF) in CArG boxes of actin promoters. In addition, myocardin-stimulated Smad2 phosphorylation and RhoA expression, leading to increased expression of type 1 collagen in an actin cytoskeleton-dependent manner. Myocardin also directly enhanced SRF expression and stimulated collagen $1 a 1$ and $1 a 2$ promoter activities. In addition, overexpression of myocardin in vivo during carbon tetrachloride-induced liver injury led to increased HSC activation and fibrogenesis. In summary, our data suggest that myocardin plays a critical role in actin cytoskeletal dynamics during HSC activation, in turn, specifically regulating type I collagen expression in hepatic myofibroblasts.

Laboratory Investigation (2017) 97, 1412-1426; doi:10.1038/labinvest.2017.96; published online 16 October 2017

The family of actins are cytoskeletal proteins that have a critical role in a wide array of cellular processes including cell division, migration, contraction, and differentiation. ${ }^{1-4}$ Thus, altered expression of actin isoforms has been a focus in multiple physiological and pathological processes. ${ }^{5,6}$ Epithelial-mesenchymal transition (EMT), for example, is a critical biological process in cancer cell metastasis, in which epithelial cancer cells acquire de novo expression of smooth muscle $\alpha$ actin (SM $\alpha$-actin), a key biomarker in EMT and differentiate to a motile phenotype. ${ }^{7}$ In wound healing and fibrosis, resident fibroblasts differentiate to the activated myofibroblasts by the virtue of expression of SM $\alpha$-actin and extracellular matrix (ECM). ${ }^{8}$ In liver fibrosis, quiescent hepatic stellate cells (HSCs) differentiate to myofibroblastlike cells, also known as hepatic myofibroblasts, through activation and acquire a number of functional phenotypes related to SM $\alpha$-actin expression. $3,9,10$
The six different actin isoforms in mammalian cells are highly conserved and have remarkable amino-acid sequence homology. ${ }^{11,12}$ Progress in understanding the functional importance of the relatively minor differences between actin isoforms has been made possible by study of specific actin isoform deficient mice. Deletion of $\beta$-actin is lethal, ${ }^{13}$ whereas cytoplasmic $\gamma$-actin deficiency leads to impaired cell growth and survival. ${ }^{14}$ SM $\alpha$-actin deficiency results in a specific defect of lactation in nursing dams and decreased contractility in activated HSCs. ${ }^{3,15}$ These findings suggest that the cytoplasmic actin isoforms are essential for cell growth and survival, whereas the more restricted actins have a critical role in tissue specific functions.

Regulation of actin expression has been extensively studied, in particular, for the smooth muscle isoform in vascular smooth muscle cells. ${ }^{16}$ This body of work suggests that actins are serum response factor (SRF) target genes as their promoters contain a 10-base pair cis element CC(A/T)6GG

Department of medicine, Medical University of South Carolina, Charleston, SC, USA

Correspondence: Professor DC Rockey, Department of Medicine, Medical University of South Carolina, 96 Jonathan Lucas Dr., Charleston, SC 29425, USA.

E-mail: rockey@musc.edu

Received 12 December 2016; revised 19 June 2017; accepted 5 July 2017 
known as the CArG box ${ }^{17}$ which allows SRF binding and activates transcription. In HSCs, SM $\alpha$-actin promoter activity appears to be particularly SRF binding dependent as mutation or deletion of the both CArG-B and A boxes leads to nearly complete elimination of the promoter activity. ${ }^{18}$ In contrast, $\beta$-actin promoter activity not only requires the CArG box but also an essential CCAAT box. ${ }^{19}$ Little is known about the mechanism underlying the regulation of cytoplasmic $\gamma$-actin. In addition to CArG box for SRF binding, SRF-induced transactivation is prominently modulated by its cofactors. ${ }^{20}$ Myocardin, as a restricted expression pattern in cardiac and smooth muscle cells, ${ }^{20,21}$ has been demonstrated to be important SRF cofactors and have a critical role in cardiomyocytes and smooth muscle cell differentiation through activation of muscle specific genes. ${ }^{22,23}$ Interestingly, myocardin was identified in HSCs in an array, linking myocardin with SM $\alpha$-actin and type 1 collagen expression during HSC activation. ${ }^{24}$ Although the molecular pathways underlying the process remains elusive.

In the current study, we explored the molecular mechanisms of actin cytoskeleton regulation by myocardin during HSC activation and hepatic myofibroblast differentiation and the effect of actin cytoskeleton alteration on ECM protein type 1 collagen expression. Our results demonstrate that myocardin induces a novel actin expression pattern through differential regulation of SM $\alpha$-actin, cytoplasmic- $\gamma$-actin, and $\beta$-actin isoforms, all of which compose the activated HSC's cytoskeleton and directly link it to morphological and functional properties in activated HSCs. Further, the data indicate an indirect link to the ECM, type 1 collagen.

\section{MATERIALS AND METHODS}

\section{Animal Model and Cell Culture}

Sprague Dawley (SD) male rats (400-500 g) were purchased from Charles' River Lab and housed in MUSCs animal facility following the NIH and the IACUC guideline. Liver fibrosis was induced by carbon tetrachloride $\left(\mathrm{CCl}_{4}\right)$ (once a week for $4 \sim 6$ weeks) as described. ${ }^{25}$ HSCs were isolated by in situ enzymatic digestion of normal or fibrotic rat liver and purified by Accudenz (Accurate Chemicals, Westbury, NY) gradient as described. ${ }^{18}$ Freshly isolated HSCs were cultured in standard 199OR medium containing $10 \%$ calf serum and $10 \%$ horse serum (Invitrogen, Grand Island, NY) in a 3\% $\mathrm{CO}_{2}$ incubator at $37^{\circ} \mathrm{C}$ otherwise stated.

\section{Adenovirus and Cell Infection}

A mouse vascular form of myocardin (856 aa) and a dominant-negative myocardin (635 aa, without the activation domain) were subcloned from pcDNA3.1-myocardin plasmid construct (a gift from Dr Olson's laboratory) into a modified pDC316 shuttle vector (Microbix, Toronto) with pCMV promoter and HA (hemagglutinin) tag. The resulting pDC316-HA-myocardin and pDC316-HA dominant-negative myocardin plasmids were co-transfected with pBGHlox $\Delta$ E1,3-cre viral plasmid into 293HEK cells to generate adenovirus-HA-myocardin (Ad-myocd) and adenovirus-HA-dominant-negative myocardin (Ad-myocdDN). An empty pDC316 shuttle vector with pCMV promoter was co-transfected with $\mathrm{pBGHlox} \Delta \mathrm{E} 1,3$-cre viral plasmid into 293HEK cells to generate adenovirus control (Ad-ctr). Viral screening and purification were performed according to manufacturer's instructions (Clontech, Mountain View, CA). Fresh isolated HSCs were cultured for 2 days and then infected with adenoviral vectors (100 MOI) in standard 199OR medium for 3 days. In vivo adenovirus $\left(2.5 \times 10^{11} \mathrm{pfu}\right.$ per rat) administration was performed via portal vein injection as described before. ${ }^{26,27}$

\section{Immunoblotting}

Immunoblotting was performed as described. ${ }^{18}$ Specific signals were captured by the Syngene G-Box digital imaging system (Syngene, Frederick, MD) and quantitative data obtained from the system's software. Raw values for control samples were arbitrarily set to 1 (or $100 \%$ ), and data were presented as fold increase or percentage of the controls. Antibodies to detect SM $\alpha$-actin (1A4) and $\beta$-actin (AC15), $\alpha$ tubulin, and Rho kinase inhibitor Y-27632 were obtained from Sigma (St. Louis, MO). Antibodies to detect cytoplasmic- $\gamma$-actin isoform (AB3265) and total actin (C4), GAPDH (as a loading control) were from Millipore (Temecula, CA). Anti-type 1 collagen (COL.1) antibody was purchased from Rockland (Gilbertsville, PA). Anti-SRF, Smad2 phosphorylation (p-Smad2), total Smad2, p-Erk, and myocardin antibodies were purchased from Cell Signaling (Danvers, MA) and Novus Bio (Littleton, CO) respectively. TGF $\beta$ were purchased from Prospec-Tany TechnoGene (Ness Ziona, Israel).

\section{Immunocytochemistry and Histology}

Immunofluorescence staining was performed as described. ${ }^{15}$ In brief, HSCs were washed with PBS twice and fixed in 4\% buffered formaldehyde solution for $15 \mathrm{~min}$. Following exposure to Triton $\times 100(0.5 \%$ in PBS) for $5 \mathrm{~min}$ and then with 3\% BSA in PBS for $30 \mathrm{~min}$, cells were incubated with primary antibodies at room temperature for $1 \mathrm{~h}$. After further washing with PBS, cells were incubated with secondary antibodies for $1 \mathrm{~h}$. Finally, cells were stained with DAPI (Sigma) for $15 \mathrm{~min}$ before mounting with FluorSave solution (Calbiochem, San Diego, CA). Anti-SM $\alpha$-actin antibody (conjugated with $\mathrm{Cy} 3$ ) and anti- $\beta$-actin antibody (AC15) were purchased from Sigma. Anti-cytoplasmic- $\gamma$-actin isoform (AB3265) antibody was from Millipore. Alexa Fluor 488 phalloidin, fluophor 488 donkey anti-sheep, fluophor 555 goat anti-rabbit and mouse antibodies were obtained from Life Technology (Carlsbad, CA). Images were captured with Olympus FV10i LIV confocal microscope (the Cell \& Molecular Imaging Shared Resource, Hollings Cancer Center, Medical University of South Carolina) and Zeiss Axio Imager M2 (Molecular Morphology, Medical University of South Carolina). For histologic analysis, liver tissue was fixed in 10\% 
buffered formalin (Fisher, NJ). Picrosirius red staining was performed as before ${ }^{28}$ and collagen content (area \%) was quantitated via image-J.

\section{Plasmid Constructs, Transfection and Luciferase Activity Assay}

Rat SM $\alpha$-actin promoter and the CArG box mutants were described before. ${ }^{18} \beta$-actin (Genbank accession number: V01217.1) and cytoplasmic- $\gamma$-actin promoters (Genbank accession number: AC095876.6) were cloned from rat genomic DNA by PCR and DNA fragments were ligated into pGL3B luciferase reporter vector (Promega, Madison, WI). CArG and CCAAT boxes in the actin promoters were mutated using site mutagenesis kit (Agilent, Santa Clara, CA). Type $1 \alpha 1$ and $1 \alpha 2$ Collagen promoters were cloned from mouse genomic DNA and ligated into pGL3B luciferase reporter vector (Promega, Madison, WI). The constructs with deletions were created by PCR approach. SRF promoter luciferase reporter constructs were described as before. ${ }^{18}$ All constructs were confirmed by sequencing (Genewiz, South Plainfield, NJ). The myocardin expression construct (pcDNA3.1+myocardin) was obtained from Dr. Eric Olson's laboratory. Co-transfections were performed in activated rat HSCs with lipofectamine 2000 (Invitrogen) as described before. $^{18}$ Luciferase activity was measured with a dualluciferase assay kit (Promega, Madison, WI). All transfection experiments were performed in triplicate and repeated twice. Relative light units (RLU) after transfection with a pGL3B reporter vector were arbitrarily set to 1 and data were presented as fold increase relative to pGL3B activity as described. ${ }^{29}$ Primers used for cloning and site mutagenesis are listed in Supplementary Table 1.

\section{Cell Contraction Assay and Wound-Healing Assay}

Freshly isolated HSCs were cultured on collagen gels (Advanced Biomatrix, San Diego, CA) as described. ${ }^{3}$ Cells were exposed to adenovirus (100 MOI) on the second day of culture and incubated for 3 further days. Then fresh medium containing serum with or without endothelin-1 (ET-1, American peptide company, Sunnyvale, CA) was added. At specified times, gels were released from the plastic substratum, and gel area was measured as described. ${ }^{3}$

Cell migration was measured using a wound healing assay kit (Cell Biolabs, San Diego, CA). In brief, cells were cultured in 24-well plates containing a specific insert in each well bottom to divide cell cultures to equally. Following 2 days of culture, the insert was removed and formed a clear, sharp demarcation on the plastic substrate. Then HSCs were exposed to adenovirus (100 MOI) in standard medium. Wound closure was subsequently measured over time and the quantitated data were obtained from triplicate experiment according to manufacturer's instructions.

\section{Real-Time PCR (RT-PCR) and RNase Protection Assay (RPA)}

RNA from HSCs was isolated using Trizol reagent (Invitrogen) and 1-2 $\mu \mathrm{g}$ of total RNA was used for first-strand cDNA synthesis by SuperScript III (Invitrogen). Resulting cDNA was PCR amplified using gene-specific primers (Supplementary Table S1) and SYBR green supermix (BioRad, Hercules, CA) on a BioRad CFX Connect Real-Time PCR Detection System. For actin isoform RNA expression analysis, $5 \mu \mathrm{g}$ of total RNA was hybridized with radioactive labeled gene-specific cRNA probes using an RPA III kit (Ambion, Austin, TX) (Supplementary Figure S1) as described before. ${ }^{18}$ Specific signals for SM $\alpha$-actin, cytoplasmic- $\gamma$-actin and $\beta$-actin were captured and quantitated using a phosphoimager (Syngene G-Box digital imaging system, Syngene). Control signals were arbitrarily set to 1 or $100 \%$ and data were presented as fold increase compared with controls.

\section{Electrophoretic Mobility Shift Assay (EMSA)}

Nuclear extracts were prepared and EMSA was performed as described before. ${ }^{18}$ In brief, nuclear extracts $(10 \mu \mathrm{g})$ were incubated with ${ }^{32} \mathrm{P}$-labeled DNA probes (for probe sequences, see Supplementary materials) at room temperature for $30 \mathrm{~min}$. For supershift assays, $2 \mu \mathrm{l}$ of anti-SRF antibody (Cell Signaling) was added to the reaction and incubated for $30 \mathrm{~min}$ at room temperature prior incubation with labeled probe. DNA-protein complexes were separated by non-denature polyacrylamide gel electrophoresis and specific signals were captured using a phosphoimager (Syngene G-Box digital imaging system).

\section{Statistical Analysis}

Quantitative data were analyzed with Microsoft Excel software. Bar graphs represent mean \pm s.d. Significance $(P<0.05)$ was assessed by the Student's $t$-test.

\section{RESULTS}

Upregulation of Myocardin Tightly Associates with Increased Expression of the Actin Cytoskeleton

To explore the basic cell and molecular mechanisms underlying myocardin-mediated HSC activation and myofibroblastlike phenotype differentiation, we first examined myocardin expression in $\mathrm{CCl}_{4}$-induced liver fibrogenesis in vivo. Myocardin mRNA was significantly increased in activated HSCs from fibrotic liver compared to HSCs from normal liver (Figure 1a). Next, as myocardin localizes to the nucleus and physically forms a complex with SRF and potentiates SRF activity, ${ }^{30,31}$ we examined myocardin and SRF protein levels in the nuclear extracts of HSCs from normal and fibrotic livers. We found that myocardin and SRF were upregulated (Figure 1b), suggesting that both of them are required for HSC activation. As myocardin regulates all smooth muscle specific genes, ${ }^{32}$ we examined the correlation between myocardin and actin isoform expression. As expected, SM $\alpha$-actin was dramatically upregulated (15-16 fold), and 


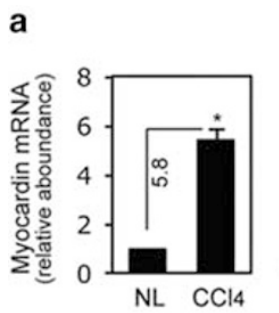

d

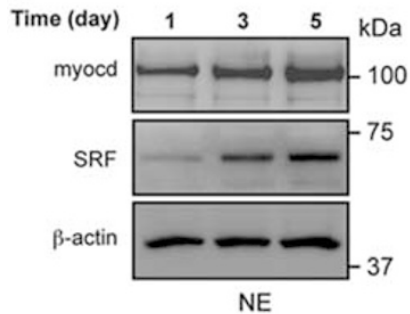

b

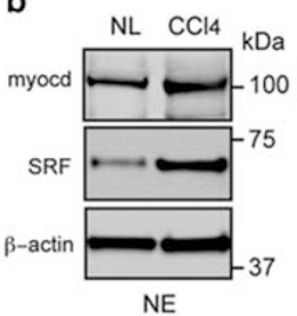

C

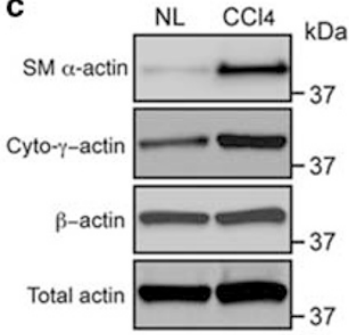

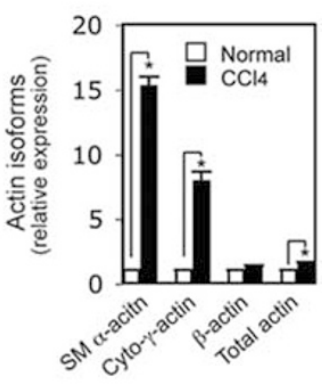

e

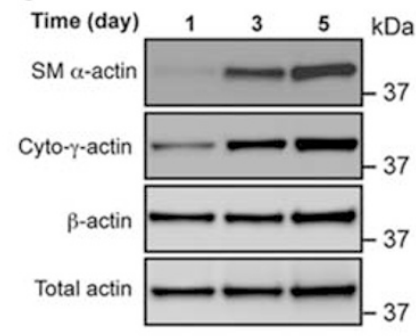

f

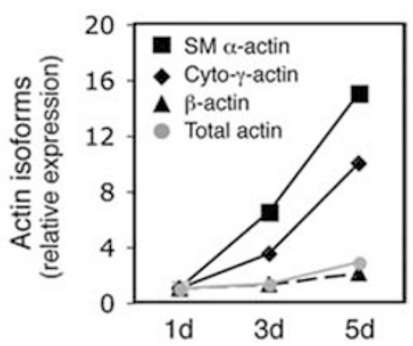

Figure 1 Myocardin is upregulated during HSC activation in vivo and in vitro. HSCs were isolated as in Methods, and in (a), total RNA from freshly isolated HSCs from normal (NL) and fibrotic rat liver $\left(\mathrm{CCl}_{4}\right)$ was extracted and subjected to RT-PCR to measure myocardin $\mathrm{mRNA}(n=3, * P<0.01$ for NL vs $\mathrm{CCl}_{4}$ ). (b) Myocardin and SRF protein levels were measured using nuclear extracts (NE) from normal (NL) and fibrotic rat liver $\mathrm{HSCs}\left(\mathrm{CCl}_{4}\right)(n=3$, ${ }^{*} P<0.05$ for $\mathrm{NL}_{\text {vs }} \mathrm{CCl}_{4}$ ). (c) Actin isoform protein profiles were measured in whole cell lysates from normal (NL) or fibrotic rat liver $\left.\mathrm{HSCs}(\mathrm{CCl})_{4}\right)$. (d) $\mathrm{HSCs}$ from normal liver were isolated and grown in culture as in methods and myocardin and SRF levels were measured in nuclear extracts; whole cell lysates were used to assess complete actin isoform profiles (e), and quantitative data are shown graphically (f). $\beta$-actin was used as a loading control (an additional Coomassie blue stain is shown in Supplementary Figure S3). Representative immunoblots from three independent experiments were shown.

surprisingly, cytoplasmic- $\gamma$-actin was also significantly elevated (7-8 fold). Unexpectedly, $\beta$-actin, a typical 'housekeeping' gene, was also increased (Figure 1c). Further, we examined whether the expression patterns of these proteins in an in vivo liver injury model recapitulated the culture-based in vitro model. Indeed, expression patterns of myocardin, SRF, SM $\alpha$-actin, cytoplasmic- $\gamma$-actin, and $\beta$-actin in cultureinduced HSC activation (Figure $1 \mathrm{~d}-\mathrm{f}$ ) were similar to that in vivo liver injury (Figure $1 \mathrm{~b}$ and $\mathrm{c}$ ). These data strongly suggest that HSC activation is associated with upregulation of myocardin and SRF, which promote actin isoform expression and actin dynamics in response to cellular differentiation signals.

\section{Myocardin Stimulates a HSC Myofibroblast-Like Phenotypic Differentiation in vitro and in vivo}

To evaluate the role of myocardin in HSC activation, primary rat HSCs were used to examine bona fide biological responses to myocardin. In addition, an adenovirus harboring a smooth muscle form of myocardin (Ad-myocd) and dominantnegative myocardin (Ad-myocd-DN) were used to modulate myocardin activity, as HSCs primarily express a smooth muscle form of myocardin (Supplementary Figure S2). HSCs infected with Ad-myocd virus exhibited a more prominent activated appearance-characterized by an enlarged cell size and prominent lamellipodia. In contrast, HSCs infected with dominant-negative myocardin had a less-activated appearance, characterized by smaller cell size and less-prominent cellular processes (Figure 2a). Myocardin-induced prominent actin stress fibers, in particular SM $\alpha$-actin stress fibers (Figure 2b) and cytoplasmic- $\gamma$-actin stress fibers (Figure 2c), which were thick and long in the cell cytoplasm. In addition, cytoplasmic- $\gamma$-actin stress fibers appeared to be prominent in filopodia around the membrane (Figure 2c). In contrast, inhibition of myocardin (with the dominant-negative construct) appeared to inhibit the activated phenotype-leading to smaller and rounder cells, with stress fibers forming a thick ring (which was mainly composed of SM $\alpha$-actin and cytoplasmic- $\gamma$-actin stress fibers) at the inside of the cell membrane and a disorganized intracellular pattern (Figure $2 \mathrm{~b}$ and $c$, lower panel). Dominant-negative myocardin also caused corresponding changes in $\beta$-actin, characterized by actin spots along thin actin stress fibers (Figure 2d). In addition, myocardin was localized in both the cytoplasm and nuclei of HSCs, whereas dominant-negative myocardin was primarily localized in the nuclei of HSCs (Figure 2e).

Further, we examined the effect of overexpression of myocardin in $\mathrm{CCl}_{4}$-induced liver fibrogenesis in vivo (Figure 3). As the myocardin-deficient mouse is an embryonic lethal and thus could not be used to examine the effect of myocardin in vivo, we chose to overexpress myocardin in HSCs with an adenovirus-expressing myocardin (note that we have previously demonstrated that parenteral administration of adenovirus leads to efficient viral expression in HSCs). ${ }^{27}$ Liver injury was established by administration of $\mathrm{CCl}_{4}$ before administration of adenovirus (Figure $3 \mathrm{a}$ and d). ${ }^{27}$ In the first 
a

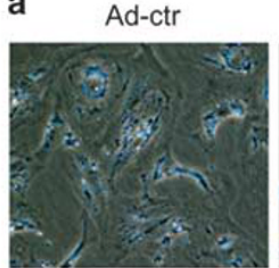

Ad-myocd

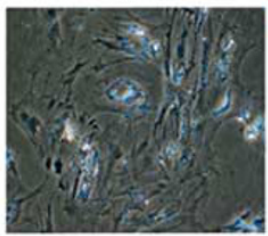

Ad-myocd-DN

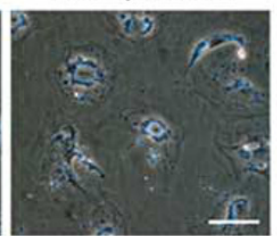

b

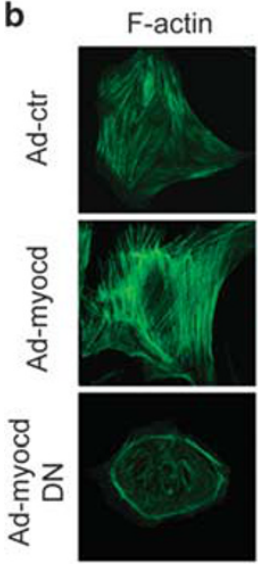

d

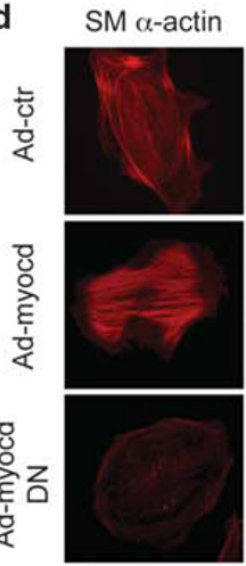

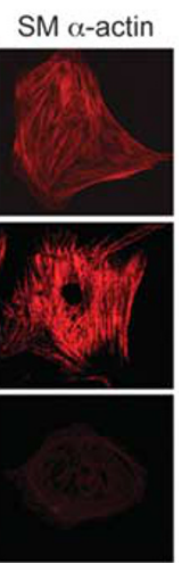

$\beta$-actin

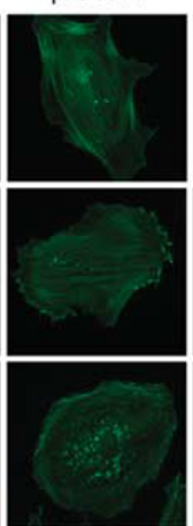

Dapi

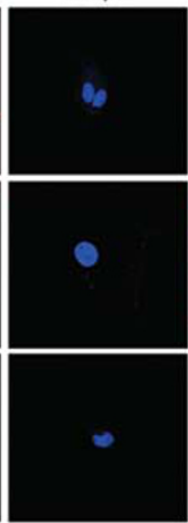

\section{Dapi}

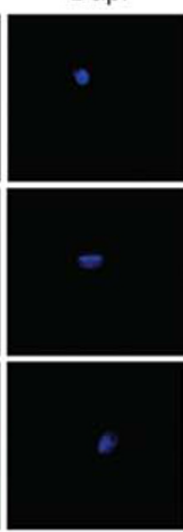

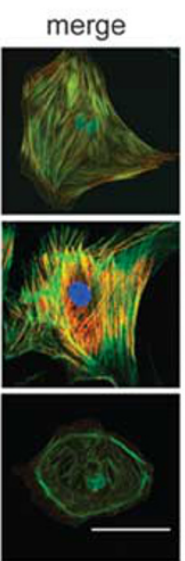

merge

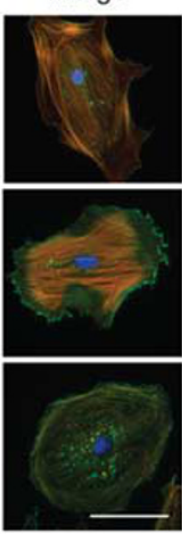

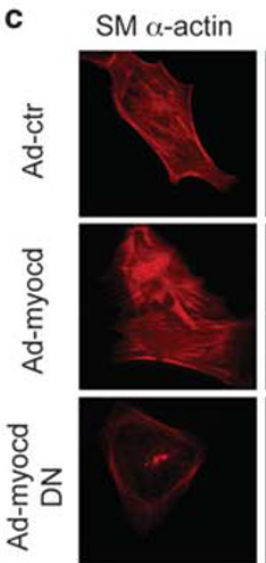

e

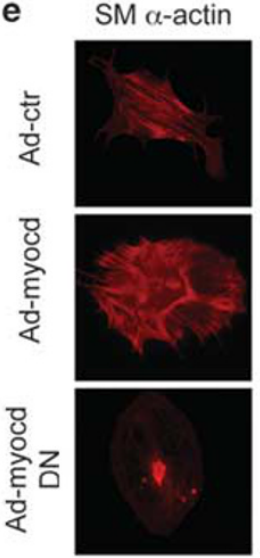

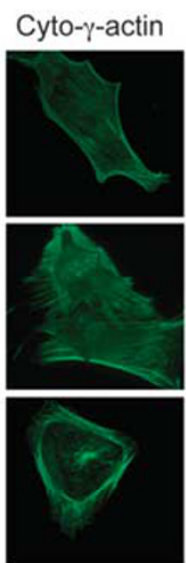

HA
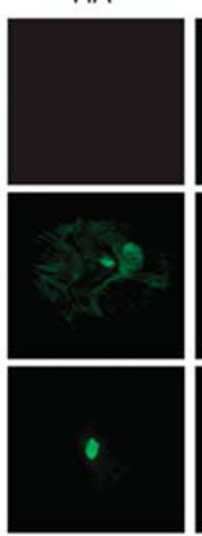
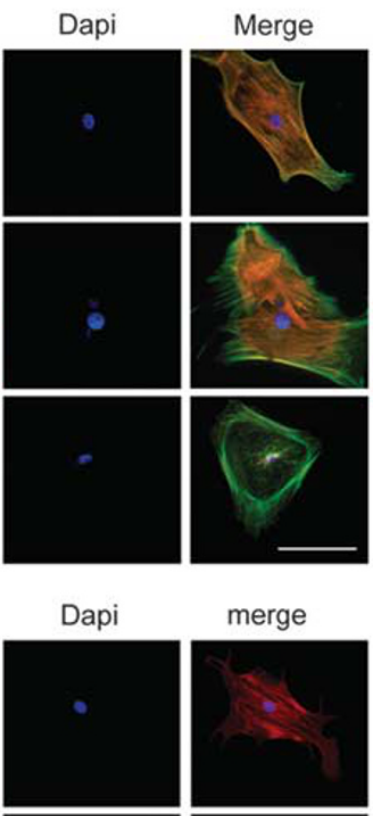

merge
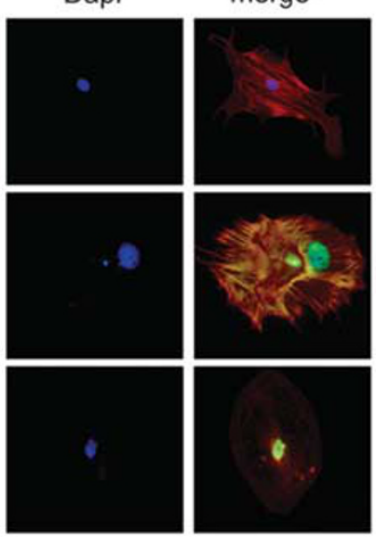

Figure 2 Myocardin induces morphological changes in activated HSCs. (a) HSCs were exposed to adenoviruses (Ad-control (Ad-ctr), Ad-myocd and Ad-myocd-DN, all at $100 \mathrm{MOI}$ ) for 3 days and then incubated at $0.5 \%$ serum medium for 2 days. Representative images from three independent experiments are shown. (b, $\mathbf{c}, \mathbf{d}, \mathbf{e})$ Cells were grown on glass cover slips and exposed to adenovirus as in (a) and subjected to immunofluorescence labeling as in Methods. Representative images from three independent experiments are shown. The scale bar represents $50 \mu \mathrm{m}$.

model, myocardin expression led to a highly activated HSC appearance and increased SM $\alpha$-actin and type $1 \alpha 1$ mRNA expression compared with control (Figure $3 \mathrm{a}-\mathrm{c}$ ). In the second $\mathrm{CCl}_{4}$-induced liver fibrosis model, ECM protein collagen deposition was increased after myocardin overexpression (compared with control, Figure $3 \mathrm{~d}$ and e). These data demonstrate the importance of myocardin in regulation of hepatic myofibroblast differentiation in vitro and in vivo.

\section{Myocardin Mediates Functional Effects in HSCs}

Prominent functional features of activated HSCs include enhanced contractility and cell motility. ${ }^{3}$ Thus, we examined whether myocardin had functional effects in HSCs. As expected, overexpression of myocardin increased basal HSC contractility, whereas inhibition of myocardin reduced ET-1mediated HSC contractility (Figure 4a-c). In addition, inhibition of myocardin activity reduced HSC migration (Figure 4d), a phenotype similar to SM $\alpha$-actin-deficient HSCs. ${ }^{3}$ Notably, exogenous expression of myocardin had minimal effects on cell motility. Taken together, the data demonstrate that myocardin has important functional effects in activated HSCs.

\section{Myocardin Differentially Regulates Actin Isoform Expression}

To explore the molecular basis of myocardin-mediated morphological and functional effects on HSCs, we examined actin isoform expression following exogenous expression of 
a

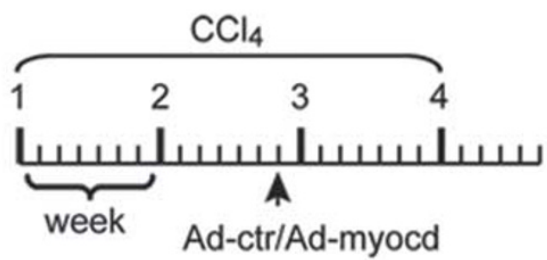

$\mathrm{CCl}_{4}+\mathrm{Ad}-\mathrm{ctr}$

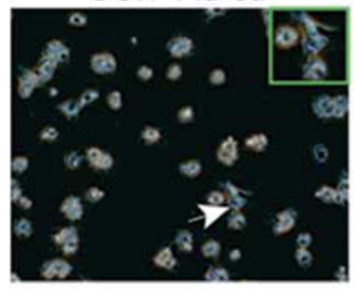

d

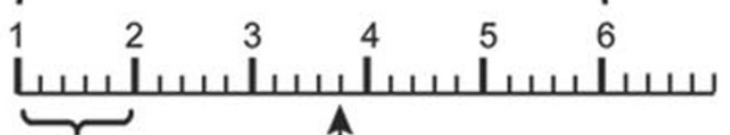

5 days

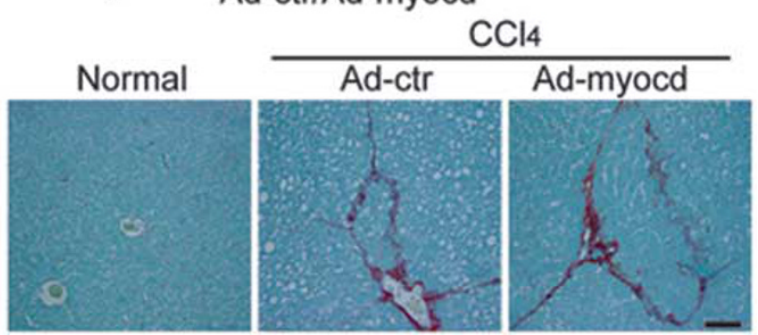

b

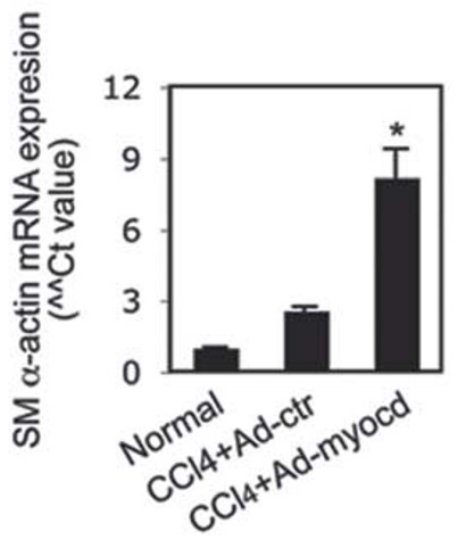

c

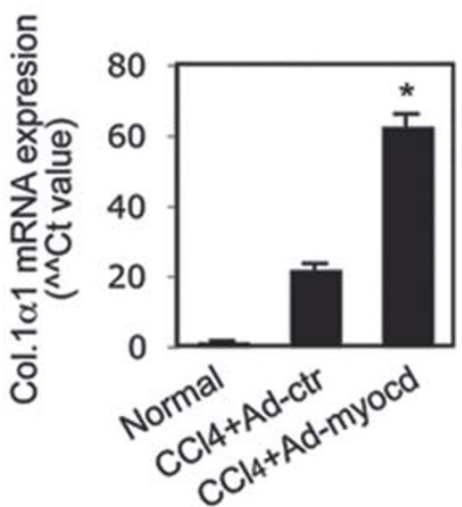

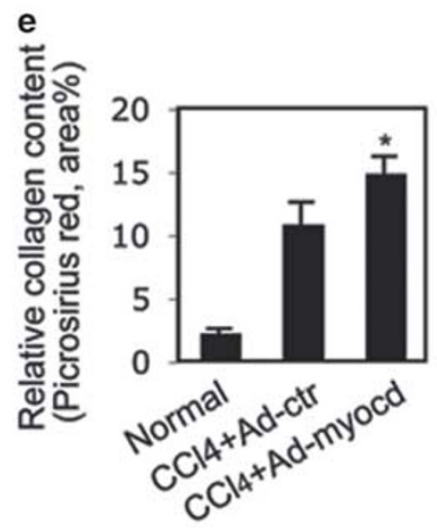

Figure 3 Overexpression of myocardin promotes HSC myofibroblast differentiation and liver fibrosis. (a) A schematic map for overexpression of myocardin with four doses of $\mathrm{CCl}_{4}$. Five days after the final dose of $\mathrm{CCl}_{4}, \mathrm{HSCs}$ were isolated and cultured overnight; representative phase contrast images are shown (arrow indicates an activated HSC). In the upper right corner of each photomicrograph is a magnified image of the area corresponding to the arrow. (b, c) Total RNA was extracted from the same cells and RT-PCR was performed to detect SM $a$-actin and Col.1a1 mRNA ( $n=3, P<0.01$ for Ad-control (Ad-ctr) vs Ad-myocd). (d) A schematic map for overexpression of myocardin with six doses of $\mathrm{CCl}_{4}$. Five days after the final $\mathrm{CCl}_{4}$ dose, whole liver tissues were harvested and subjected to picrosirius red staining as in Methods (representative images are shown in (d) and in (e) quantitative data are depicted graphically ( $n=3 \sim 4, P<0.05$ for Ad-control (Ad-ctr) vs Ad-myocd). The scale bar represents $50 \mu \mathrm{m}$.

myocardin or dominant-negative myocardin in activated HSCs. To measure the bona fide target actin isoform mRNA expression, we used unique probes specific to nucleotides in the amino termini of the actins (with RPA, Supplementary Figure S1). SM $\alpha$-actin and cytoplasmic- $\gamma$-actin mRNA levels were upregulated by myocardin (6-7 fold and almost twofold $v s$ control, respectively) (Figure $5 \mathrm{a}$ and $\mathrm{b}$ ). $\beta$-actin mRNA was also increased compared with the control. In contrast, inhibition of myocardin led to significant decreases in SM $\alpha$-actin and cytoplasmic- $\gamma$-actin mRNAs, whereas $\beta$-actin mRNA was only modestly affected (Figure $5 \mathrm{a}$ and $\mathrm{c}$ ).

Next, we examined regulation of actin protein isoforms by myocardin. Overexpression of myocardin caused dramatic upregulation of SM $\alpha$-actin. Cytoplasmic- $\gamma$-actin and $\beta$-actin were also significantly increased (7-8 fold and $0.8-1$ fold $v$ s control, respectively), which all composed of the increased total actin (Figure $5 \mathrm{~d}$ and e). These results were similar to the actin expression patterns in activated HSCs induced by culture or in vivo liver injury (Figure 1c,e and f), suggesting a bona fide biological effect of myocardin on actin isoform expression. In contrast, after blocking myocardin function (Ad-myocd-DN), both SM $\alpha$-actin and cytoplasmic- $\gamma$-actin were significantly decreased (by $78 \%, 56 \%$, respectively); $\beta$ actin was also reduced, which all together led to $>50 \%$ reduction in the total actin (Figure $5 \mathrm{~d}$ and $\mathrm{f}$ ). In aggregate, these data suggest that myocardin differentially regulates actin isoform expression, which appears to be primarily via transcriptional regulation.

\section{CArG box and ATTA, CCAAT Boxes are Specifically Required for Myocardin-Induced Differential Regulation of Actin Isoform Transcription}

The CArG box, found in the promoter region of many genes coding for structural proteins, has been established as the core 
a

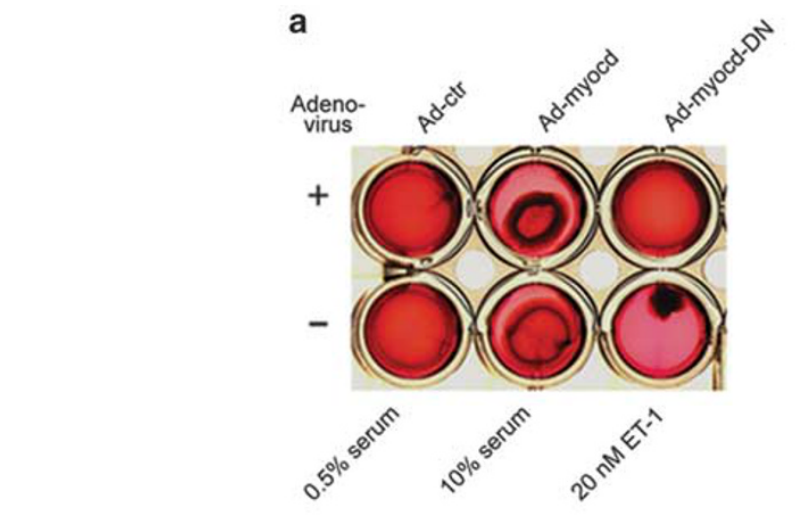

b

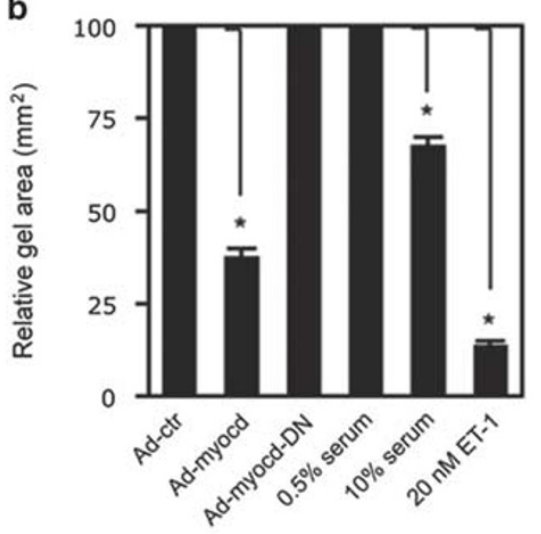

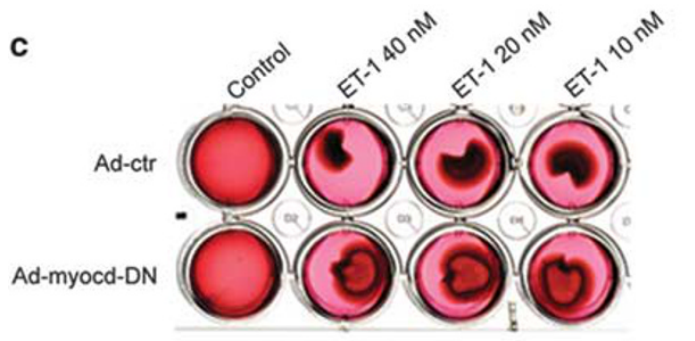

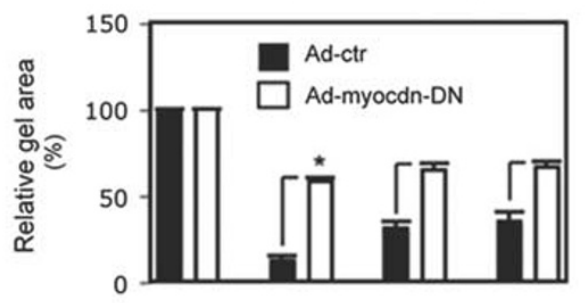

d
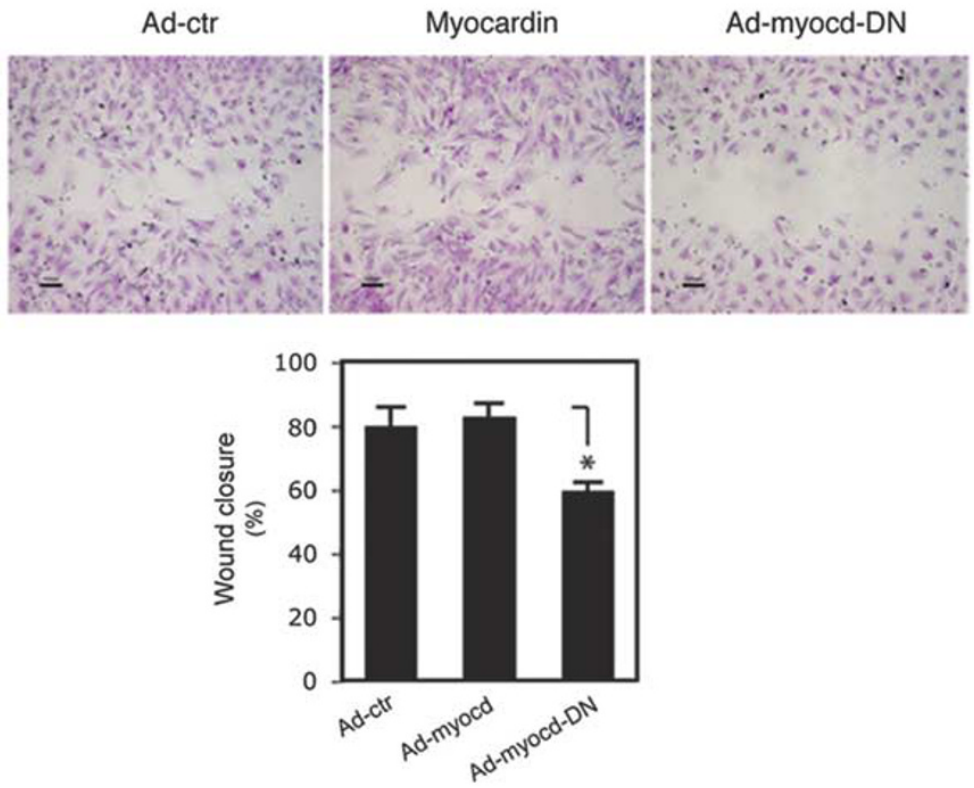

Figure 4 Myocardin-mediated functional effects. HSCs were isolated and grown on collagen lattices for 2 days and exposed to adenovirus as indicated for 3 days. Lattice contraction was as in Methods and images were taken $12 \mathrm{~h}$ after gel lattice release; (a) $10 \%$ serum and $20 \mathrm{nM}$ ET-1 were used as positive controls and $0.5 \%$ serum as a negative control. A representative image from 3 independent experiments is shown. In (b), quantitative data are presented graphically (the stronger the contraction, the smaller the gel area. $n=3,{ }^{*} P<0.05$ for Ad-control (Ad-ctr) vs Ad-myocd, $10 \%$ serum and $20 \mathrm{nM}$ ET-1, respectively). (c) Cells were isolated, grown in $0.5 \%$ serum containing medium, and exposed to different concentrations of ET-1 as indicated at the time of lattice release; images were taken after gel lattice release ( $6 \mathrm{~h}$ and a representative image from 3 independent experiments is shown). In the panel below the image, quantitative data are shown ( $n=3,{ }^{*} P<0.05$ for Ad-control (Ad-ctr) vs Ad-myocd-DN). (d) HSCs were grown in a $24-w e l l$ woundhealing assay plate for 2 days and then exposed to the indicated adenovirus at the same time as application of the scratch wound (images were taken after a further 3 days of culture and representative images are shown). In the graph below the images, the area of wound closure was measured as in Methods and quantitative data are shown graphically $\left(n=3,{ }^{*} P<0.05\right.$ for Ad-control (Ad-ctr) vs Ad-myocd-DN).

SRF-binding sequence. ${ }^{16,17}$ We next examined whether CArG boxes in the different actin promoters (Figure 6a) might have differential activity in HSCs. We found that SRF-binding activity in the SM $\alpha$-actin promoter was lower than in the cytoplasmic- $\gamma$-actin or $\beta$-actin promoter at baseline (Figure 6b, black columns 1 vs 5 and 9). However, myocardin potently stimulated SRF binding to the SM $\alpha$-actin promoter, which was greater than for the cytoplasmic- $\gamma$-actin or $\beta$-actin promoter (Figure 6b, open columns 2 vs 1, 6 vs 5 and 10 vs 9). Mutation of CArG boxes (Figure 6a) in the SM $\alpha$-actin promoter nearly eliminated SM $\alpha$-actin promoter activity and almost completely abrogated myocardin-induced promoter activity (Figure 6b, columns 3 and 4 ). In contrast, mutation of CArG boxes in cytoplasmic- $\gamma$-actin or $\beta$-actin promoters had a less-prominent effect, particularly in the cytoplasmic- $\gamma$-actin promoter. CArG box mutation had a substantial effect on both cytoplasmic- $\gamma$-actin and $\beta$-actin promoter activities with myocardin stimulation (Figure 6b, columns 7 vs 5, 8 vs 6 and 11 vs 9,12 vs 10). These data indicate that the CArG boxes in actin promoters are required for basal and myocardininduced transcriptional activation, but that there is a differential effect for the different actin CArG boxes. 

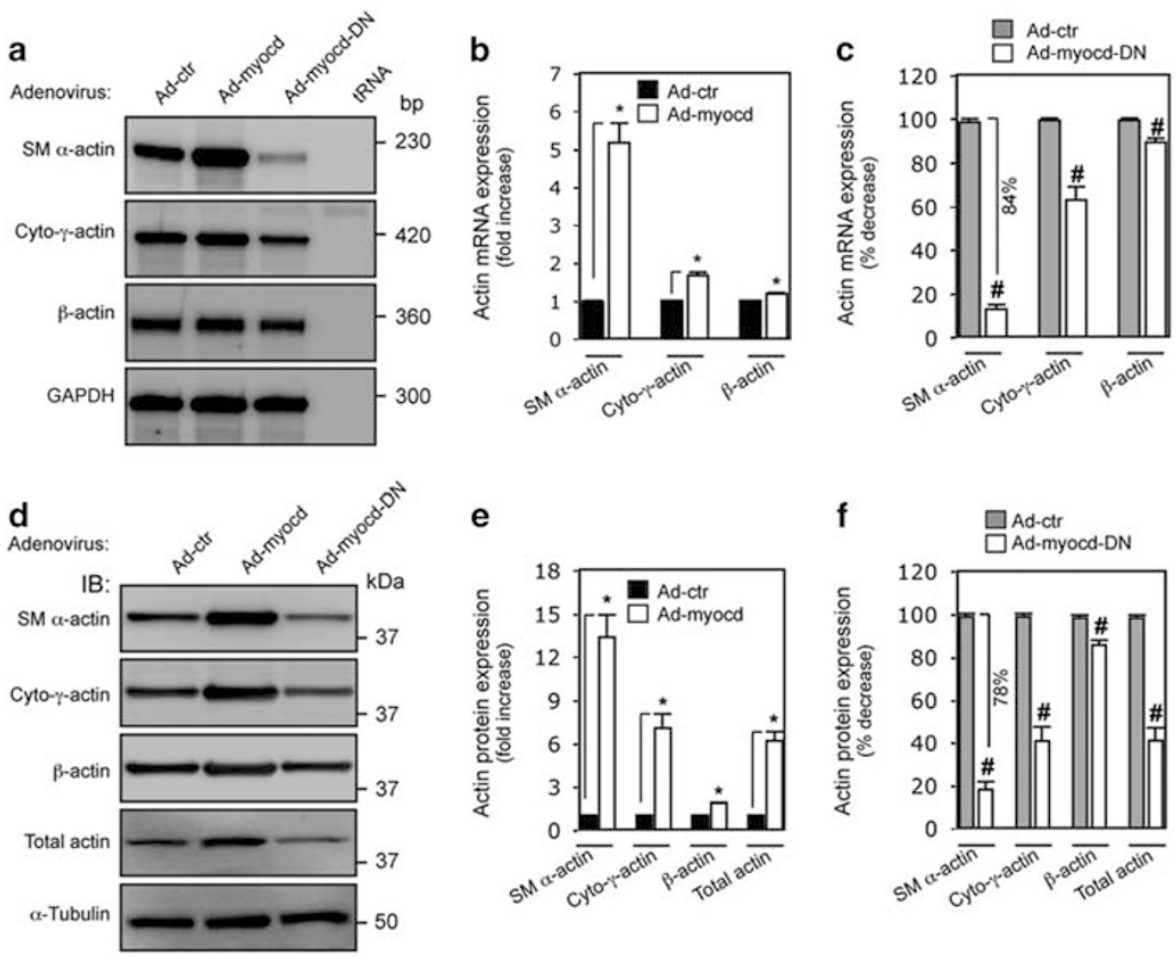

Figure 5 Myocardin induces differential expression of actin isoforms. HSCs were isolated as above and exposed to adenovirus as indicated for 3 days and then incubated in $0.5 \%$ serum medium for 2 days. (a) Actin isoform mRNA profiles were assessed by RNase protection assay (RPA). Representative images from three independent experiments are shown. (b) Quantitative data depicting changes in actin isoform mRNA expression induced by myocardin, or dominant-negative myocardin (c) are depicted graphically. (d) Whole HSC lysates were subjected to immunoblotting to analyze actin isoform profiles (representative immunoblots from three independent experiments are shown) and (e) quantitative data depicting changes in actin isoform expression induced by myocardin, or dominant-negative myocardin (f) are presented graphically. ( $n=4,{ }^{*} P<0.05$ for $\mathrm{Ad}$-control (Ad-ctr) vs Admyocd; $\# P<0.05$ for Ad-control vs Ad-myocd-DN).

We further examined whether SRF-binding activity could be associated with the observed differential (myocardinmediated) actin promoter activities. As expected, SRF-binding activity to all of the actin CArG boxes was increased in nuclear extracts after expression of myocardin (Figure $6 c$, lane $3 v s$ lane 2), indicating that myocardin enhanced SRF-binding activity, consistent with previous data in smooth muscle cells. ${ }^{33}$ Interestingly, SRF-binding activity to the CArG boxes in the SM $\alpha$-actin promoter was significantly reduced by a dominant-negative myocardin construct (Figure $6 c$, panel 1 and 2; lane 4 vs lane 2), but SRF-binding activity to CArG boxes in cytoplasmic- $\gamma$-actin and $\beta$-actin promoters were minimally affected (Figure 6c, panel 3, 4; lane $4 v s$ lane 2). We hypothesized that such differential SRF-binding activity among the CArG boxes might be due to different $\mathrm{A} / \mathrm{T}$ content, as CArG boxes in the SM $\alpha$-actin promoter contain single $\mathrm{C}$ or $\mathrm{G}$ substitutions (Figure 6a). By using identical amounts of nuclear extract from activated HSCs (Figure 6d, lower panel) and the same amount of probe harboring individual CArG box sequences (Figure 6d), we found that the SM $\alpha$-actin CArG-B box exhibited lower SRF-binding activity (Figure 6d, lane 2) than cytoplasmic $\beta$ - or $\gamma$-actin
CArG boxes (Figure 6d, lane 4). Interestingly, although the CArG boxes in cytoplasmic- $\gamma$-actin and $\beta$-actin promoters are both conserved (Figure 6a), more SRF-binding activity was present in the CArG box of cytoplasmic- $\gamma$-actin than in the $\beta$-actin CArG box (Figure 6d, lane 4 vs 3). Taken together, these results revealed that CArG boxes with different $\mathrm{A} / \mathrm{T}$ content led to differential SRF binding. Further, the SM $\alpha$-actin promoter appeared to be more sensitive to myocardin stimulation than the other two actin promoters, suggesting a reverse relationship between SRF binding and myocardin sensitivity.

Previous studies revealed that a conserved ATTA box in SM $\alpha$-actin promoter ${ }^{33}$ and a CCAAT box ${ }^{19}$ in the cytoplasmic $\beta$ actin promoter play an important role in actin mRNA expression. Therefore, we examined whether these boxes have functional effects on myocardin-induced promoter activity among the actin isoforms (Figure 6e). Myocardin-induced SM $\alpha$-actin promoter activity was prominently reduced in the ATTA box mutant construct (1.9 fold reduction) compared with the wild-type construct (Figure 6f, column 4 vs 2). Similarly, the CCAAT box mutation significantly abrogated myocardin-induced cytoplasmic- $\gamma$-actin and $\beta$-actin 
a

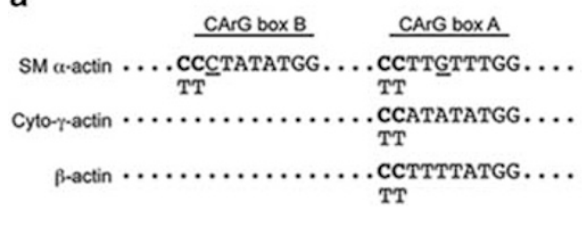

C

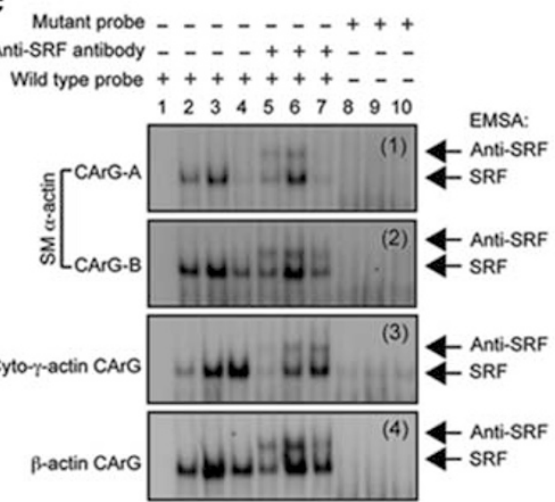

e

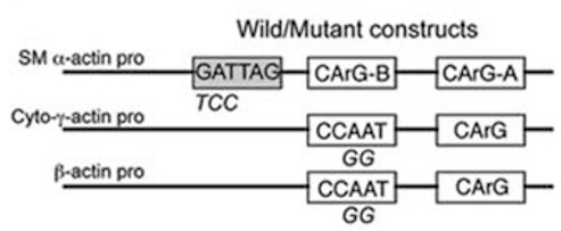

g

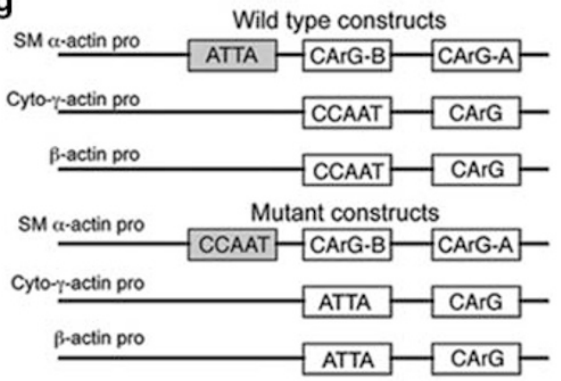

b

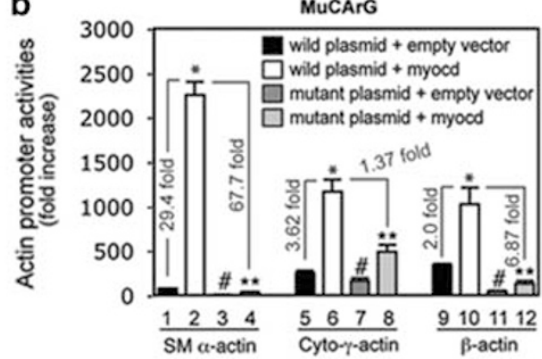

d
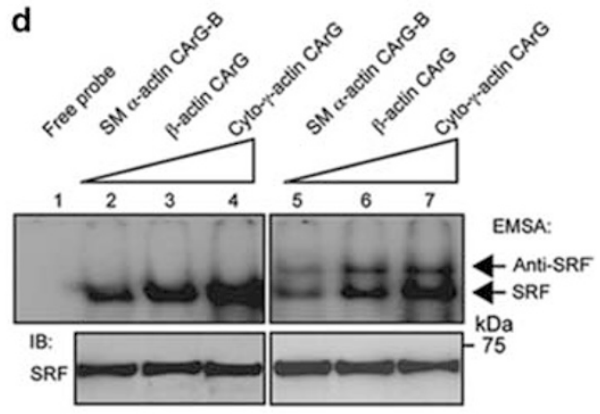

$\mathbf{f}$

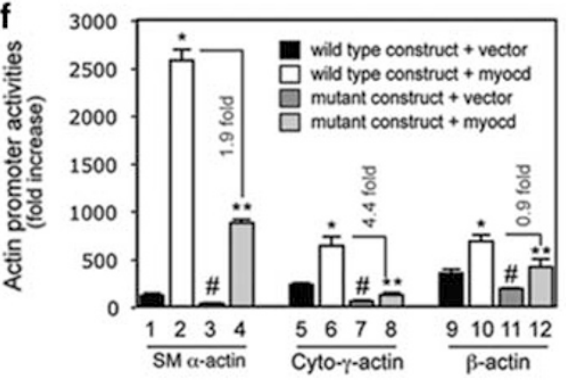

h

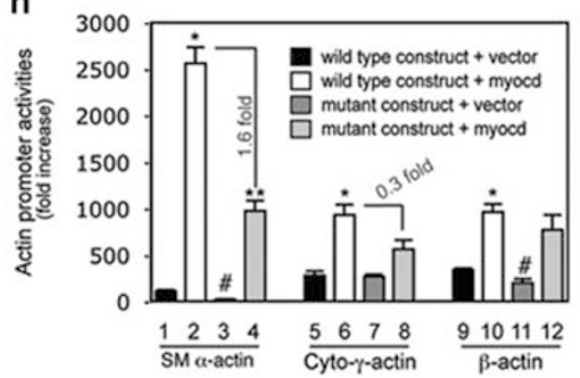

Figure 6 Myocardin-induced differential regulation of actin isoforms is CArG box and ATTA, CCAAT box dependent. (a) CArG box sequences from rat SM $\alpha$-actin, cytoplasmic- $\gamma$-actin (Cyto- $\gamma$-actin), and $\beta$-actin promoters are aligned. Site mutations are shown in bold letters (CC in CArG boxes were replaced by TT). (b) Activated HSCs were co-transfected with luciferase reporter constructs containing wild type or the CArG box mutation as in a and a myocardin expression plasmid (Myocd) or empty vector. Cells were harvested 2 days later to detect for promoter activity. (c) After growth for 2 days after isolation, HSCs were exposed to the indicated adenoviral vectors for 3 days and 2 days later, they were subjected to EMSA to measure the effect of myocardin on SRF-binding activity. SRF binding and supershifted bands are highlighted by arrows (lane 1, 2, 5, 8: nuclear extracts from HSCs infected with Ad-control virus; lane 3, 6, 9: nuclear extracts from HSCs infected with Ad-myocd virus; lane 4, 7, 10: nuclear extracts from HSCs infected with Admyocd-DN virus). Representative data from three independent experiments are shown. (d) EMSA was performed using nuclear extracts (10 $\mu \mathrm{g})$ from activated HSCs and the same amount of different actin CArG box probes $\left(1 \times 10^{5} \mathrm{cpm}\right)$ as indicated. SRF binding and supershifted complexes were indicated by arrows (upper panel). Nuclear extracts were probed by anti-SRF antibody as loading control (bottom panel). Representative data from three independent experiments are shown. (e) A schematic diagram of wild type and mutant actin isoform promoters is shown (mutated nucleotides were indicated below the consensus sequences); (f) luciferase assays were performed as in $\mathbf{b}$. (g) A schematic diagram of exchanged elements (ATTA and CCAAT boxes) among SM $a$-actin, cytoplasmic- $\gamma$-actin (cyto- $\gamma$-actin), and $\beta$-actin promoters is shown; (h) luciferase activity assay was performed as in $\mathbf{b}$. ( $n=3,{ }^{*} P<0.01$ for wild plasmid+empty vector vs wild plasmid+myocardin; $n=3, \# P<0.05$ for wild plasmid+empty vector vs mutant plasmid+empty vector; $n=3,{ }^{* *} P<0.05$ for wild plasmid+myocardin vs mutant plasmid+myocardin). 
promoter activity (4.4 and 0.9 fold reduction, respectively) (Figure 6f, columns $8 v s$ 6; 12 vs 10). These results suggest that the ATTA (SM $\alpha$-actin promoter) and CCAAT boxes (cytoplasmic- $\gamma$-actin and $\beta$-actin promoter) are required for full myocardin-mediated transactivation.

Next, we examined whether the ATTA box was able to stimulate cytoplasmic- $\gamma$-actin and $\beta$-actin promoter activity as it appeared to be critical for myocardin-induced promoter activity in the SM $\alpha$-actin promoter (Figure 6g). Interestingly, substitution of the CCAAT box in the cytoplasmic- $\gamma$-actin promoter with the ATTA box did not change the promoter activity as that in the CCAAT box mutant construct (Figure 6f), but such substitution prominently restored myocardin-induced cytoplasmic- $\gamma$-actin promoter activity (Figure $6 \mathrm{~h}$, column 8 vs 6 ). Similar to the $\beta$-actin promoter, replacement CCAAT box with the ATTA box also significantly restored myocardin-mediated promoter activity (Figure 5h, column 12 vs 10). In contrast, substitution of the ATTA box in the SM $\alpha$-actin promoter with a CCAAT box led to the same result as that in the ATTA box mutant SM $\alpha$-actin promoter construct (Figure 6 h, column 4 vs 2). These results suggest that the ATTA box is an indispensable element in SM $\alpha$-actin promoter (ie, to mediate myocardin-induced full promoter activity). However, the CCAAT box is specifically required for myocardin-induced cytoplasmic- $\gamma$ actin and $\beta$-actin actin promoter activity.

\section{Myocardin Mediates type 1 Collagen Expression in HSCs}

Type 1 collagen expression is regulated via multiple factors, including MRTF/A. ${ }^{34}$ Myocardin, as a major member in this family, ${ }^{35}$ may also have a role in regulation of type 1 collagen expression in HSCs. Indeed, type 1 collagen expression was increased by wild-type myocardin but decreased by dominant-negative myocardin (Figure 7a). To explore the molecular pathways underlying this process, we examined the effect of myocardin on TGF $\beta$-induced type 1 collagen expression. As expected, TGF $\beta$ enhanced type 1 collagen expression in control HSCs. SM $\alpha$-actin and cytoplasmic $\gamma$ actin were also upregulated by TGF $\beta$. However, TGF $\beta$ induced type 1 collagen expression was abrogated by dominant-negative myocardin, which was accompanied by reduced expression of actin isoforms (Figure $7 \mathrm{~b}$ ). In addition, myocardin prominently enhanced Smad2 phosphorylation, but dominant-negative myocardin did not significantly reduce Smad2 phosphorylation compared with the control (Figure $7 \mathrm{c}$ ). The results suggest that myocardin is able to stimulate TGF $\beta$ signaling through myocardin-mediated actin expression, thereby regulates type 1 collagen expression. ${ }^{36}$

We next examined the role of myocardin in ET-1-mediated type 1 collagen expression, ET- 1 also increases type 1 collagen expression (via Erk and AP-1 pathways ${ }^{37}$ ). Similar to TGF $\beta$, ET-1 upregulated type 1 collagen expression, accompanied by increased expression of SM $\alpha$-actin, cytoplasmic $\gamma$-actin, SRF, and Erk phosphorylation. These effects were prominently inhibited by dominant-negative myocardin (Figure 7d), suggesting that myocardin is able to regulate type 1 collagen expression via ET-1-mediated actin cytoskeleton proteins, SRF, and Erk pathways.

Finally, as Rho signaling mediates actin stress fiber formation and disruption of Rho pathway suppresses collagen accumulation in activated HSCs, ${ }^{38}$ we examined the effect of Y-27632, a specific Rho kinase inhibitor, on myocardininduced type 1 collagen expression. As expected, Y-27632 disrupted actin stress fibers and led to a disorganized actin cytoskeleton in control HSCs (Figure 7e). The effect of Y-27632 was abrogated by exogenous expression of myocardin (Figure 7e, lower panel). Type 1 collagen expression was significantly decreased by Y-27632 in control HSCs, but myocardin rescued the effect of Y-27632 (Figure 7f, top panel). Simultaneously, Rho inhibition resulted in decreased SM $\alpha$-actin and cytoplasmic $\gamma$-actin expression, and was also rescued by myocardin. These findings provide further support for the concept that myocardin mediates actin and actin dependent regulation of type 1 collagen expression.

\section{Myocardin Targets SRF Expression and Stimulates Type 1 Collagen Transcription}

In activated HSCs, as the SRF gene promoter has CArG boxes, ${ }^{18,39}$ and as upregulation of myocardin coincided with increased SRF expression (Figure $1 \mathrm{~b}$ and $\mathrm{d}$ ), we postulated that myocardin might directly regulate SRF. Indeed, exogenous expression of myocardin increased both SRF mRNA and SRF protein levels in HSCs, whereas dominant-negative myocardin decreased SRF mRNA and protein expression (Figure $8 \mathrm{a}$ and $\mathrm{b}$ ). To further examine the transcriptional regulation of SRF, wild type (WT) and CArG mutant SRF promoter constructs were co-transfected with myocardin expression plasmid in activated HSCs. ${ }^{18}$ Myocardin dramatically upregulated SRF promoter activity (8.8 fold increase), whereas myocardin failed to fully elevate SRF promoter activity in the CArG box mutant SRF promoter construct (Figure 8c). The data suggest that myocardin positively stimulates an SRF autoregulatory loop.

As the type $1 \alpha 1$ and $1 \alpha 2$ collagen promoters also contain a CArG box, ${ }^{40}$ we also examined whether myocardin might potentiate type $1 \alpha 1$ and $1 \alpha 2$ collagen promoter activity. Exogenous expression of myocardin led to a significant increase in both type $1 \alpha 1$ and $1 \alpha 2$ collagen promoter activities (Figure 8d and e), whereas the myocardin-induced effect was inhibited by mutation of the CArG box in both promoters. These data suggest that myocardin may directly activate type $1 \alpha 1$ and $1 \alpha 2$ collagen transcription via SRF.

\section{DISCUSSION}

A defining property of the HSC to myofibroblast differentiation is the de novo expression of SM $\alpha$-actin, which associates with multiple functional features of activated HSCs, ${ }^{9}$ although the fundamental molecular mechanisms underlying hepatic myofibroblast differentiation remain poorly understood. In the present study, our results demonstrate that myocardin is 
an important factor to promote HSC activation process. Myocardin positively targets SRF autoregulatory loop to increase SRF expression. Myocardin and SRF appear to serve as a core regulatory platform to differentially regulate actin isoform expression through myocardin's different sensitivity to differential SRF-binding activity on the CArG boxes as well as the ATTA and CCAAT boxes in different actin promoters. Myocardin-induced upregulation of actin expression not only contributes to morphological and functional features in activated HSCs, but also indirectly regulates type 1 collagen expression (Figure 8f).

HSC activation toward myofibroblast differentiation is a complicated molecular process in which multiple factors are involved, including MRTF/ $/ \mathrm{A}^{34}$ and myocardin (Figure 1). Our results suggest that HSCs may have unique myofibroblastic features as HSCs contain vitamin A lipid droplets ${ }^{10}$
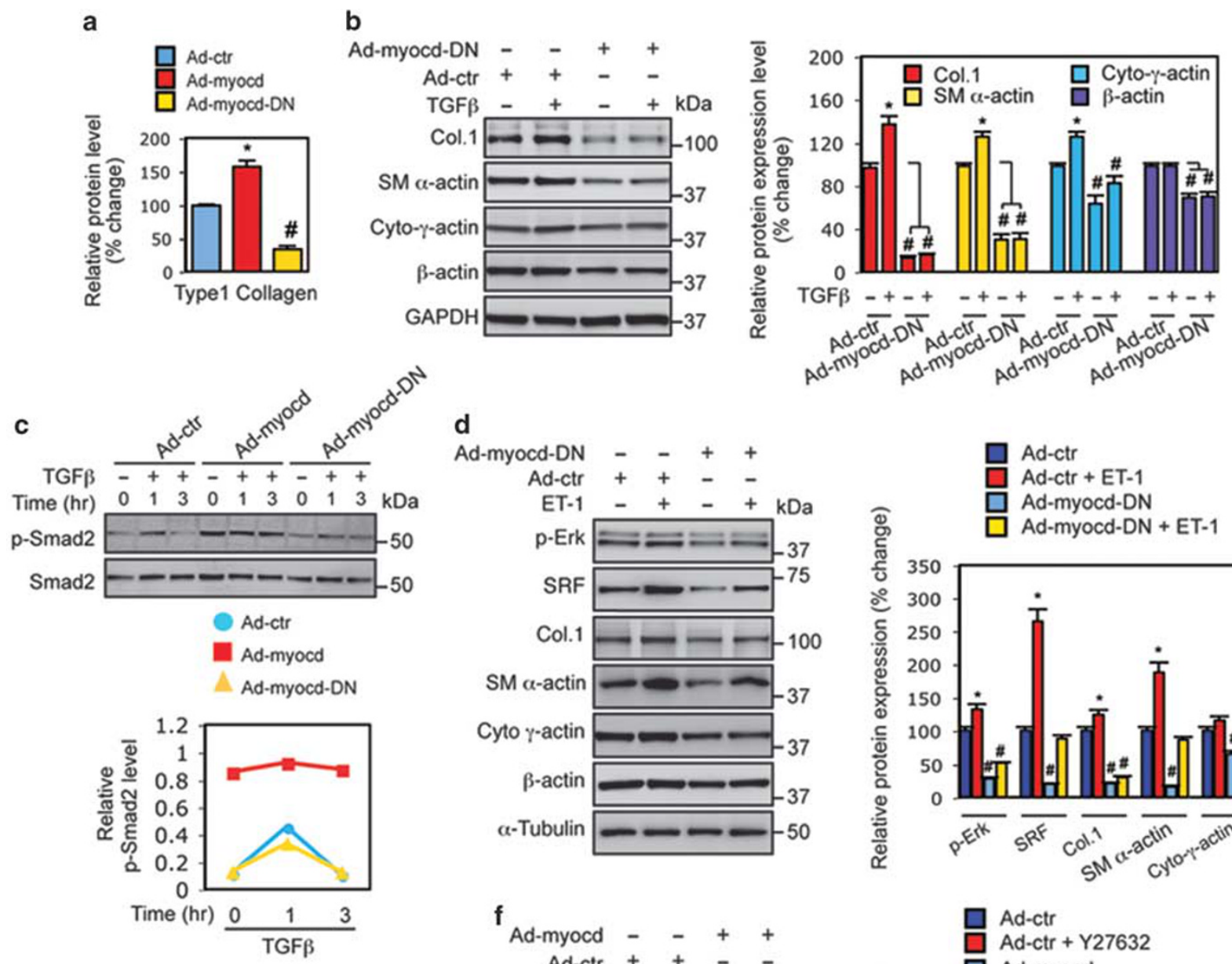

d
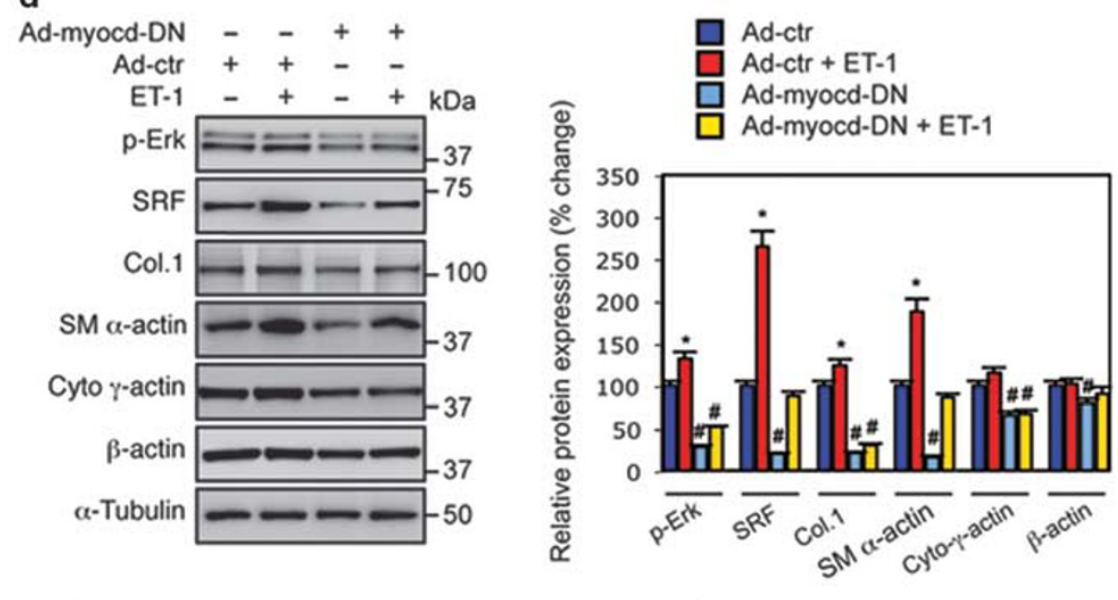

e
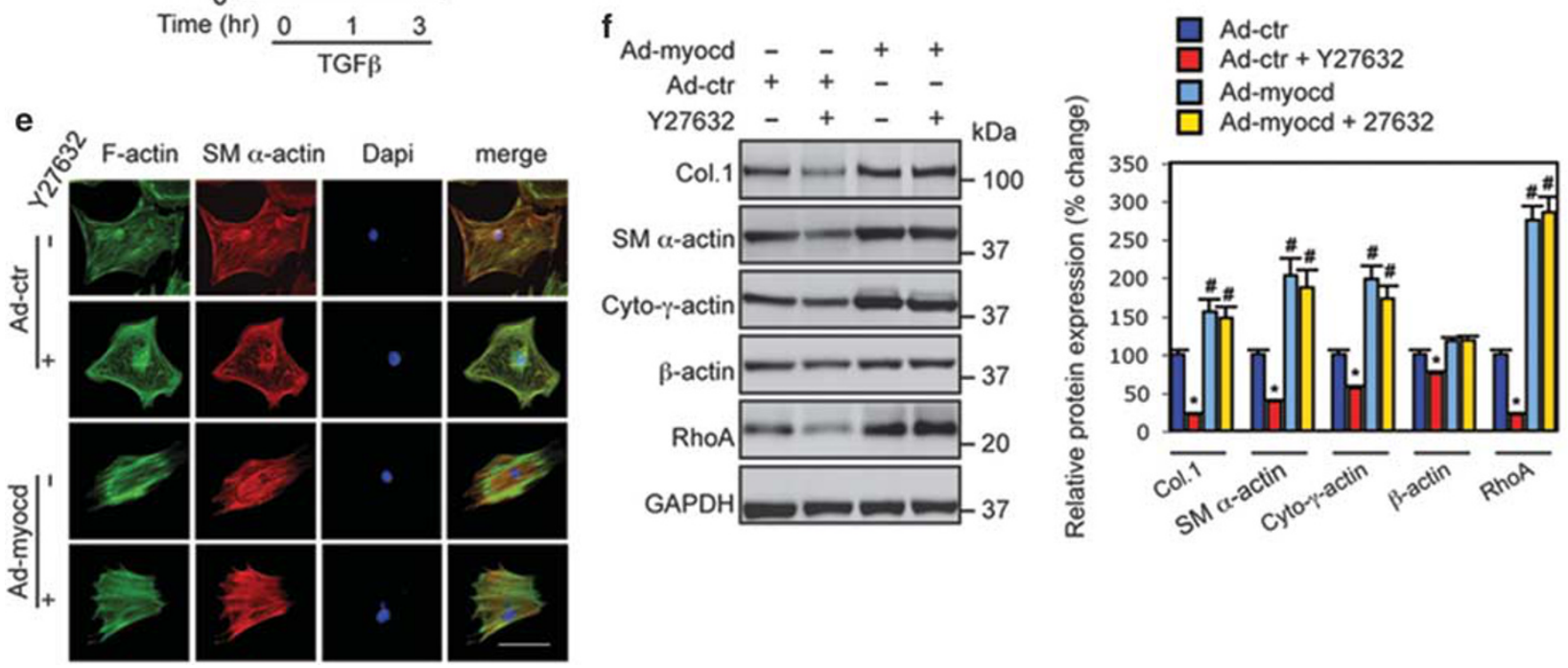
and express myocardin (Figure 1), ${ }^{24,41}$ which are different from the myofibroblasts of other tissue sources such as myofibroblasts in kidney and lung fibrosis, ${ }^{42,43}$ suggesting that different mechanisms may exist among different tissue types during wound-healing process. Myocardin is located primarily in the nucleus (Figure 2$)^{35}$ and readily activates gene transcription via interaction with SRF. In contrast, MRTF/A is an actin binding protein and its nuclear translocation requires the release from actin binding in response to serum stimulation and other signals that promote actin polymerization. ${ }^{20}$ It is likely that myocardin stimulates HSC activation via upregulation of smooth muscle specific genes, whereas MRTF/A may potentiate the effect of myocardin in response to increased actin polymerization during HSC activation and myofibroblast differentiation.

SM $\alpha$-actin is a well-known molecular marker for myofibroblast differentiation. ${ }^{8-10}$ Our results revealed that in addition to SM $\alpha$-actin, cytoplasmic $\gamma$-actin was significantly upregulated in activated HSCs (Figure 1). The finding suggests that cytoplasmic $\gamma$-actin may also have an important role in promotion of HSC activation, which was not reported in previous studies regarding to HSC activation. Noticeably, cytoplasmic $\beta$-actin was also increased, which together with SM $\alpha$-actin and cytoplasmic $\gamma$-actin consisted of the total actin pool in activated HSCs. We also found that myocardin enhanced actin stress fiber formation (Figure 2), presumably due to increased RhoA expression induced by myocardin (Figure 7f). Taken together, increased expression of actin isoforms and actin stress fibers are likely critical for morphological and functional attributes of hepatic myofibroblasts (Figures 2-4).

The molecular mechanism underlying differential regulation of the actin isoforms during HSC activation remains poorly understood. Our results revealed that an opposite phenomenon between myocardin-induced actin promoter activity and the SRF-binding activity to the CArG box of the actin promoters-the higher promoter activity was induced by myocardin with the lower SRF-binding activity. The nature under this phenomenon was largely due to the $\mathrm{A} / \mathrm{T}$ content and maybe the ratio of $\mathrm{A}$ to $\mathrm{T}$ in the CArG boxes. Our findings were consistent with a previous study in smooth muscle cells, ${ }^{44}$ in which the conserved c-fos CArG box had more SRF-binding activity than the CArG boxes of SM $\alpha$ actin promoter, whereas myocardin-induced promoter activity was prominently reduced in the CArG boxes of $\operatorname{SM} \alpha$-actin promoter with c-fos CArG box substitution. Noticeably, our results also revealed that conserved cis elements - ATTA in the SM $\alpha$-actin promoter and CCAAT box in the cytoplasmic $\gamma$-I $\beta$-actin actin promoters mediated myocardin-induced actin promoter activity as mutation of these conserved elements prominently damped myocardin-induced promoter activity in all three actin promoter activity (Figure 6e and f). Thus, the CArG-A and B boxes as well as the ATTA box provided myocardin-induced complete SM $\alpha$-actin promoter activity. Unlike SM $\alpha$-actin as a myocardin regulatory gene was reported in previous studies, ${ }^{32}$ myocardin-induced upregulation of cytoplasmic $\gamma$-actin and $\beta$-actin were not previously described. Our results showed that the CCAAT box was likely more important than the CArG box for cytoplasmic $\gamma$-actin promoter activity, whereas the CArG box appeared to be more important than the CCAAT box for $\beta$-actin promoter activity (Figure $6 \mathrm{~b}$ and e). Interestingly, myocardin-induced promoter activity between cytoplasmic $\gamma$-actin and $\beta$-actin promoters was similar (Figure 6b), but more prominently increased expression of cytoplasmic $\gamma$-actin mRNA and protein was observed compared to $\beta$-actin (Figures 1 and $5)$. Our data highlight the complicated nature of myocardininduced regulation of actin isoforms and further raise the possibility that posttranscriptional regulation might be important in actin isoform expression in activated HSCs.

Previous investigation revealed that MRTF/A was involved in regulation of type 1 collagen expression in liver wound healing. ${ }^{34}$ Our results demonstrated that myocardin also plays an important role in regulation of type 1 collagen expression in activated HSCs in vitro (Figure 7) and in vivo (Figure 3 ). TGF $\beta$ signaling is critical to mediate type 1 collagen

Figure 7 Myocardin modulates type 1 collagen expression. (a) HSCs were exposed to adenoviruses as indicated for 3 days and then incubated in $0.5 \%$ serum medium for 2 further days. Type 1 collagen (Col.1) was measured by immunoblotting and quantitative data are shown ( $n=3$, ${ }^{*}<0.01$ for Adcontrol (Ad-ctr) vs Ad-myocd; $\# P<0.05$ for Ad-ctr vs Ad-myocd-DN). (b) HSCs were exposed to Ad-control or Ad-myocd-DN for 3 days and then incubated in $0.5 \%$ serum medium with or without TGF $\beta$ (10 ng) for 2 further days. Type 1 collagen and actin isoform profiling were performed by immunoblotting. Representative images from three independent experiments were shown and quantitative data are shown in the graph to the right ( $n=3,{ }^{*} P<0.05$ for Ad-control (Ad-ctr) vs Ad-ctr + TGF $\beta$; $\# P<0.05$ for Ad-ctr vs Ad-myocd-DN with or without TGF $\beta$ ). (c) HSCs were as in b; HSCs were exposed to TGF $\beta$ (10 ng) for 0,1 , and $3 \mathrm{~h}$, respectively. Whole cell lysates were assayed for phospho-Smad2 ( $\mathrm{p}$-Smad2) and total Smad2 as indicated. Representative immunoblots from three independent experiments are shown. Quantitative data are shown in the graph below. (d) HSCs were as in b, and were exposed to ET-1 (20 nM) for 2 days; cell lysates were assayed for p-Erk, SRF, Col.1, and actin isoform by immunoblotting; representative images from three independent experiments are shown and quantitative data are shown in the graph to the right $(n=3, * P<0.05$ for Ad-control (Adctr) vs Ad-ctr + ET-1; \#P<0.05 for Ad-ctr vs Ad-myocd-DN with or without ET-1). (e, f) HSCs were isolated and grown on either glass cover slips as in Figure (2B) or standard culture dishes as above. Following virus infection for 3 days, cells were incubated in $0.5 \%$ serum medium with or without Y-27632 (10 $\mu \mathrm{M})$ for 1 day. Cells on glass cover slips were fixed and immunostained as in Methods and whole cell lysates from the same cell preparations were subjected to immunoblotting to detect type 1 collagen, RhoA and actin isoforms (left panel). Representative images from three independent experiments are shown and quantitative data are shown in the graph to the right ( $n=3, * P<0.05$ for Ad-control (Ad-ctr) vs Ad-ctr with Y-27632; $\# P<0.05$ for Ad-ctr vs Ad-myocd with or without Y-27632). The scale bar represents $50 \mu \mathrm{m}$. 

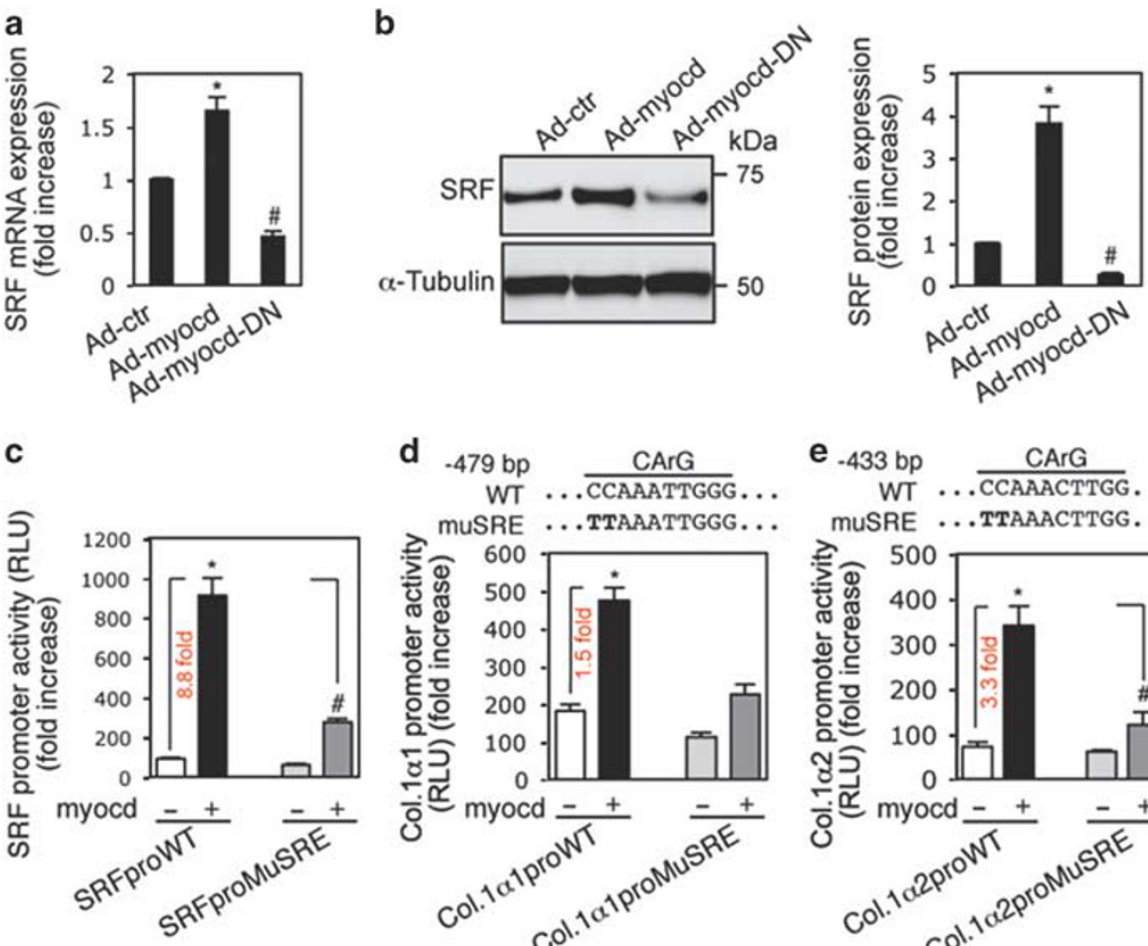

d

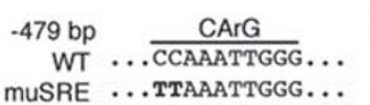
e -433 bp CArG
WT ... $\overline{\text { CCAAACTTGG }}$...
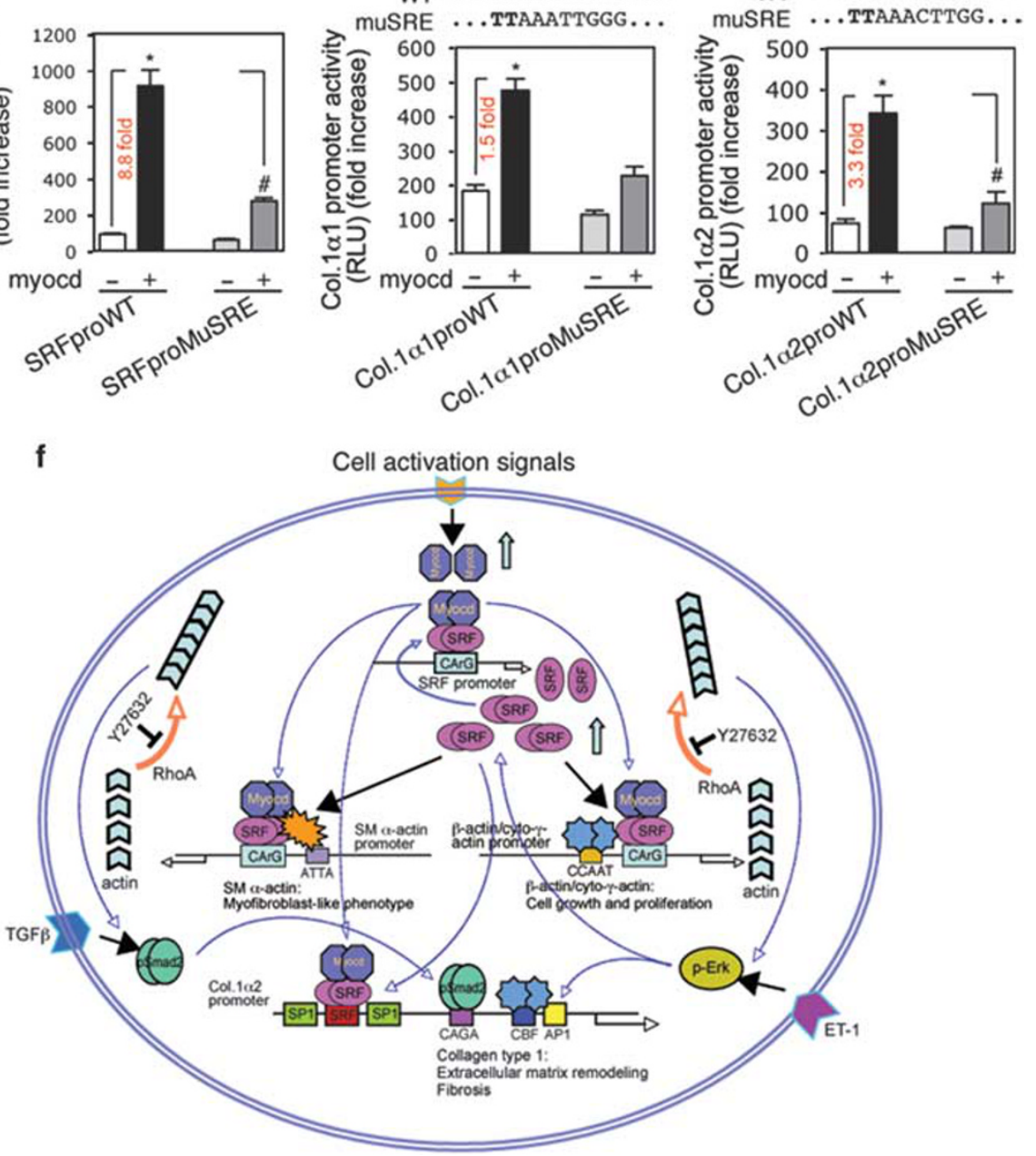

Figure 8 Myocardin activates SRF and type 1 collagen transcription via CArG boxes in actin promoters. HSCs were cultured and infected with adenoviruses as in Figure 7a. (a) SRF mRNA expression was measured by RT-PCR and (b) SRF was measured by immunoblotting; representative images from 3 independent experiments are shown and quantitative data are shown in the graph to the right ( $n=5$, ${ }^{*} P<0.01$ for Ad-control (Ad-ctr) vs Admyocd; $\# P<0.05$ for Ad-ctr vs Ad-myocd-DN). (c) Activated HSCs were co-transfected with a wild type (WT) SRF promoter (SRFproWT) or CArG box mutant SRF promoter (SRFproMuSRE) and a myocardin expression plasmid (myocd) as in Figure 6b. (d, e) Activated HSCs were co-transfected with wild type $1 a 1$ and $1 a 2$ collagen promoters (Col.1a1proWT, Col.1a2proWT) or CArG box mutant type $1 a 1$ and 2 collagen promoters (Col.1a1proMuSRE, Col.1a2proMuSRE) and a myocardin expression plasmid as in Figure $6 \mathrm{~b}$. Quantitative data are depicted graphically $\left(n=3,{ }^{*} P<0.01\right.$ for WT construct with empty vector vs with myocardin expression plasmid; \#P<0.01 for WT construct with myocardin expression plasmid vs SRE mutant construct with a myocardin expression plasmid). (f) A schematic diagram providing a conceptual overview of myocardin-mediated hepatic stellate cell activation and myofibroblast differentiation is shown. 
expression in various fibrotic conditions. ${ }^{45}$ Unlike MRTF/A, which did not increase $\mathrm{p}$-Smad2, ${ }^{46}$ our data revealed that myocardin prominently elevated p-Smad2 level (Figure 7c), suggesting that myocardin was able to signal to the TGF $\beta$ pathway. Notably, a dominant-negative myocardin decreased type 1 collagen expression and all three actin isoforms without an effect on p-Smad2, suggesting an indirect effect of TGF $\beta$ signaling through myocardin-mediated actin expression (Figure $7 \mathrm{~b}$ and $\mathrm{c}$ ). Furthermore, our results suggested that myocardin was also able to affect ET-1 signaling via regulating Erk phosphorylation and SRF expression (Figure 7d), which is another important pathway in type 1 collagen expression as COL1 $\alpha 2$ promoter has both AP-1 and SRF-binding sites. ${ }^{47}$

The Rho pathway has an important role in actin polymerization. ${ }^{5,36}$ Our results indicated that myocardin stimulates RhoA expression (Figure 7f), which is likely the molecular mechanism for myocardin-induced actin stress fiber formation (Figures 2 and 7e) and functional features such as enhanced contractility in HSCs (Figure 4). ${ }^{36}$ Indeed, basal Rho activity was required to maintain a normal actin dynamics and type 1 collagen expression, which was documented by suppression of Rho signaling with Y-27632 (Figure $7 \mathrm{e}$ and f). However, exogenous expression of myocardin upregulated type 1 collagen production and all actin isoforms as well as RhoA levels in presence of Y-27632, suggesting a feedback pathway from myocardin to RhoA via actin and actin polymerization. ${ }^{36}$ It is likely that myocardininduced Rho/actin signals may be resulted from heterodimerization with MRTF-A through its conserved leucine zipper domain at least in part ${ }^{20}$ as HSCs express both myocardin and MRTF-A.

In addition, our results showed that myocardin was able to directly regulate type $1 \alpha 1$ and $1 \alpha 2$ collagen transactivation via CArG boxes in the promoters (Figure 8d and e). Although myocardin, as an SRF cofactor, was previously reported to regulate SRF target genes, ${ }^{23,32}$ our results for the first time showed that myocardin was able to regulate SRF expression (Figure $8 \mathrm{a}$ and $\mathrm{b}$ ), which provided a positive feedback regulatory loop from myocardin to SRF, and SRF to SRF target genes (Figure 8f).

In summary, we have demonstrated that myocardin promotes HSC activation and myofibroblast differentiation. The CArG box, together with ATTA and/or CCAAT boxes, in the actin promoters primarily mediate myocardin-induced differential expression of actin isoforms, which make up the activated HSC cytoskeleton. We further show that myocardin also stimulates type 1 collagen transcription, enhances SRF expression, which appears to be a critical mechanism to rapidly enrich SRF expression in activated HSCs. Thus, myocardin is responsible for morphological and functional changes that occur during HSC activation and myofibroblast differentiation, and raise the possibility that this pathway could be targeted therapeutically.
Supplementary Information accompanies the paper on the Laboratory Investigation website (http://www.laboratoryinvestigation.org)

\section{ACKNOWLEDGMENTS}

We thank Eric Olson (UT Southwestern, TX, USA) for myocardin expression plasmids (dominant active and negative version). We also thank Yingyu Ren for HSC preparation and Mudan Ren for assistance with immunofluorescence staining. This work was supported by NIH grant R01 DK098819.

\section{DISCLOSURE/CONFLICT OF INTEREST}

The authors declare no conflict of interest.

1. Heng YW, Koh CG. Actin cytoskeleton dynamics and the cell division cycle. Int J Biochem Cell Biol 2010;42:1622-1633.

2. Gardel ML, Schneider IC, Aratyn-Schaus Y, et al. Mechanical integration of actin and adhesion dynamics in cell migration. Annu Rev Cell Dev Biol 2010;26:315-333.

3. Rockey DC, Weymouth N, Shi Z. Smooth muscle alpha actin (Acta2) and myofibroblast function during hepatic wound healing. PLoS One 2013;8:e77166.

4. Mack CP. Signaling mechanisms that regulate smooth muscle cell differentiation. Arterioscler Thromb Vasc Biol 2011;31:1495-1505.

5. Sandbo N, Dulin N. Actin cytoskeleton in myofibroblast differentiation: ultrastructure defining form and driving function. Transl Res 2011;158: 181-196.

6. Yamaguchi H, Condeelis J. Regulation of the actin cytoskeleton in cancer cell migration and invasion. Biochim Biophys Acta 2007;1773:642-652.

7. Lamouille S, Xu J, Derynck R. Molecular mechanisms of epithelialmesenchymal transition. Nat Rev Mol Cell Biol 2014;15:178-196.

8. Hinz B, Phan SH, Thannickal VJ, et al. The myofibroblast: one function, multiple origins. Am J Pathol 2007;170:1807-1816.

9. Brenner DA, Kisseleva T, Scholten D, et al. Origin of myofibroblasts in liver fibrosis. Fibrogenesis Tissue Repair 2012;5:S17.

10. Sato M, Suzuki S, Senoo H. Hepatic stellate cells: unique characteristics in cell biology and phenotype. Cell Struct Funct 2003;28:105-112.

11. Perrin BJ, Ervasti JM. The actin gene family: function follows isoform. Cytoskeleton (Hoboken) 2010;67:630-634.

12. Bunnell TM, Ervasti JM. Structural and functional properties of the actin gene family. Crit Rev Eukaryot Gene Expr 2011;21:255-266.

13. Bunnell TM, Burbach BJ, Shimizu Y, et al. beta-Actin specifically controls cell growth, migration, and the G-actin pool. Mol Biol Cell 2011;22:4047-4058.

14. Bunnell TM, Ervasti JM. Delayed embryonic development and impaired cell growth and survival in Actg1 null mice. Cytoskeleton (Hoboken) 2010;67:564-572.

15. Weymouth N, Shi Z, Rockey DC. Smooth muscle alpha actin is specifically required for the maintenance of lactation. Dev Biol 2012;363:1-14.

16. Owens GK, Kumar MS, Wamhoff BR. Molecular regulation of vascular smooth muscle cell differentiation in development and disease. Physiol Rev 2004;84:767-801.

17. Miano JM, Long X, Fujiwara K. Serum response factor: master regulator of the actin cytoskeleton and contractile apparatus. Am J Physiol Cell Physiol 2007;292:C70-C81.

18. Shi Z, Rockey DC. Interferon-gamma-mediated inhibition of serum response factor-dependent smooth muscle-specific gene expression. J Biol Chem 2010;285:32415-32424.

19. Danilition SL, Frederickson RM, Taylor CY, et al. Transcription factor binding and spacing constraints in the human beta-actin proximal promoter. Nucleic Acids Res 1991;19:6913-6922.

20. Parmacek MS. Myocardin-related transcription factors: critical coactivators regulating cardiovascular development and adaptation. Circ Res 2007;100:633-644.

21. Huang J, Wang T, Wright AC, et al. Myocardin is required for maintenance of vascular and visceral smooth muscle homeostasis during postnatal development. Proc Natl Acad Sci USA 2015;112:4447-4452.

22. Li S, Wang DZ, Wang $Z$, et al. The serum response factor coactivator myocardin is required for vascular smooth muscle development. Proc Natl Acad Sci USA 2003;100:9366-9370.

23. Wang Z, Wang DZ, Pipes GC, et al. Myocardin is a master regulator of smooth muscle gene expression. Proc Natl Acad Sci USA 2003;100: 7129-7134. 
24. Shimada $\mathrm{H}$, Ochi $\mathrm{T}$, Imasato $\mathrm{A}$, et al. Gene expression profiling and functional assays of activated hepatic stellate cells suggest that myocardin has a role in activation. Liver Int 2010;30:42-54.

25. Rockey DC, Boyles JK, Gabbiani G, et al. Rat hepatic lipocytes express smooth muscle actin upon activation in vivo and in culture. J Submicrosc Cytol Pathol 1992;24:193-203.

26. Gerolami R, Cardoso J, Bralet MP, et al. Enhanced in vivo adenovirusmediated gene transfer to rat hepatocarcinomas by selective administration into the hepatic artery. Gene Ther 1998;5:896-904.

27. Yu Q, Que LG, Rockey DC. Adenovirus-mediated gene transfer to nonparenchymal cells in normal and injured liver. Am J Physiol Gastrointest Liver Physiol 2002;282:G565-G572.

28. Rehman H, Liu Q, Krishnasamy $\mathrm{Y}$, et al. The mitochondria-targeted antioxidant MitoQ attenuates liver fibrosis in mice. Int J Physiol Pathophysiol Pharmacol 2016;8:14-27.

29. Li T, Shi Z, Rockey DC. Preproendothelin-1 expression is negatively regulated by IFNgamma during hepatic stellate cell activation. Am J Physiol Gastrointest Liver Physiol 2012;302:G948-G957.

30. Wang D, Chang PS, Wang $Z$, et al. Activation of cardiac gene expression by myocardin, a transcriptional cofactor for serum response factor. Cell 2001;105:851-862.

31. Mack CP, Hinson JS. Regulation of smooth muscle differentiation by the myocardin family of serum response factor co-factors. J Thromb Haemost 2005:3:1976-1984.

32. Du KL, Ip HS, Li J, et al. Myocardin is a critical serum response factor cofactor in the transcriptional program regulating smooth muscle cell differentiation. Mol Cell Biol 2003;23:2425-2437.

33. Yoshida T, Hoofnagle MH, Owens GK. Myocardin and Prx1 contribute to angiotensin Il-induced expression of smooth muscle alpha-actin. Circ Res 2004;94:1075-1082.

34. Tian W, Hao C, Fan $Z$, et al. Myocardin related transcription factor $A$ programs epigenetic activation of hepatic stellate cells. J Hepatol 2015;62:165-174.

35. Pipes GC, Creemers EE, Olson EN. The myocardin family of transcriptional coactivators: versatile regulators of cell growth, migration, and myogenesis. Genes Dev 2006;20:1545-1556.
36. Olson EN, Nordheim A. Linking actin dynamics and gene transcription to drive cellular motile functions. Nat Rev Mol Cell Biol 2010;11:353-365.

37. Davis J, Molkentin JD. Myofibroblasts: trust your heart and let fate decide. J Mol Cell Cardiol 2014;70:9-18.

38. Murata T, Arii S, Nakamura T, et al. Inhibitory effect of Y-27632, a ROCK inhibitor, on progression of rat liver fibrosis in association with inactivation of hepatic stellate cells. J Hepatol 2001;35:474-481.

39. Belaguli NS, Schildmeyer LA, Schwartz RJ. Organization and myogenic restricted expression of the murine serum response factor gene. A role for autoregulation. J Biol Chem 1997;272:18222-18231.

40. Luchsinger LL, Patenaude CA, Smith BD, et al. Myocardin-related transcription factor-A complexes activate type I collagen expression in lung fibroblasts. J Biol Chem 2011;286:44116-44125.

41. Wirz W, Antoine M, Tag CG, et al. Hepatic stellate cells display a functional vascular smooth muscle cell phenotype in a threedimensional co-culture model with endothelial cells. Differentiation 2008;76:784-794

42. Sun $\mathrm{KH}$, Chang $\mathrm{Y}$, Reed $\mathrm{NI}$, et al. alpha-Smooth muscle actin is an inconsistent marker of fibroblasts responsible for force-dependent TGFbeta activation or collagen production across multiple models of organ fibrosis. Am J Physiol Lung Cell Mol Physiol 2016;310:L824-L836.

43. Takeji M, Moriyama T, Oseto $S$, et al. Smooth muscle alpha-actin deficiency in myofibroblasts leads to enhanced renal tissue fibrosis. J Biol Chem 2006;281:40193-40200.

44. Hendrix JA, Wamhoff BR, McDonald OG, et al. 5' CArG degeneracy in smooth muscle alpha-actin is required for injury-induced gene suppression in vivo. J Clin Invest 2005;115:418-427.

45. Leask A, Abraham DJ. TGF-beta signaling and the fibrotic response. FASEB J 2004;18:816-827.

46. Velasquez LS, Sutherland LB, Liu Z, et al. Activation of MRTF-Adependent gene expression with a small molecule promotes myofibroblast differentiation and wound healing. Proc Natl Acad Sci USA 2013;110:16850-16855.

47. Small EM, Thatcher JE, Sutherland LB, et al. Myocardin-related transcription factor-a controls myofibroblast activation and fibrosis in response to myocardial infarction. Circ Res 2010;107:294-304. 